\title{
EFECTO DE LA TRANSICIÓN AL MECANISMO PLÁSTICO EN LA INESTABILIDAD DINÁMICA.
}

\author{
Gerardo Díaz Martínez ${ }^{(\mathbf{1})}$ y Emilio Sordo Zabay ${ }^{(2)}$
}

\begin{abstract}
RESUMEN
Se evalúa la importancia de la transición del estado elástico al plástico de estructuras sometidas a análisis dinámicos, enfocándose a determinar si la formación paulatina de articulaciones plásticas en la estructura afecta al coeficiente de colapso dinámico $(C c)$. Para ello, se estudia un conjunto de marcos planos y sistemas de un grado de libertad trilineales sometidos a la acción de registros acelerométricos y se analiza su comportamiento cercano al colapso por inestabilidad dinámica. Los registros utilizados se clasifican por el tipo de suelo de la estación y por la distancia epicentral. Se observan diferencias de hasta un $50 \%$ en el $C c$, entre marcos con las mismas propiedades elásticas, cortante resistente y forma del mecanismo plástico, donde la variable a considerar es la transición elasto-plástica. Por otro lado, se obtienen espectros de colapso para sistemas de un grado de libertad con ciclo histerético trilineal sometidos al mismo grupo de registros. De los resultados obtenidos se puede concluir que los sistemas con comportamiento bilineal muestran valores del coeficiente de colapso más conservadores que los sistemas con histéresis trilineal.
\end{abstract}

Palabras clave: inestabilidad dinámica; mecanismo plástico; coeficiente de estabilidad

\begin{abstract}
This paper focuses on the variations of the dynamic collapse coefficient $(C c)$ produced by differences in the characteristics of mechanism development. The $C c$ is thus obtained firstly for a group of frames having in common elastic properties, ultimate base shear strength and collapse mechanism when subjected to different ground motions, then, for a group of single degree of freedom systems. Records are classified by soil type and epicentral distance. Results for frames show differences close to $50 \%$ in $C c$, depending on the mechanism development characteristics. On the other hand, collapse spectra are computed for trilinear single degree of freedom systems subjected to the same set of records. From these spectra, it can be concluded that systems with bilinear behaviour always lead to conservative values of the dynamic collapse coefficient.
\end{abstract}

Key words: dynamic instability; plastic mechanism; instability index

Artículo recibido el 24 de marzo de 2010 y aprobado para su publicación el 26 de septiembre de 2011

(1) Estudiante de Posgrado en Ingeniería Estructural, Universidad Autónoma Metropolitana Azcapotzalco, Av. San Pablo 180, Col. Reynosa Tamaulipas, 02200, México D.F., email: diazmgerardo@hotmail.com

(2) Profesor, Departamento de Materiales Universidad Autónoma Metropolitana Azcapotzalco, Av. San Pablo 180 , Col. Reynosa Tamaulipas, 02200, México D.F., email: esz@correo.azc.uam.mx 


\section{INTRODUCCIÓN}

Aunque prevenir el colapso es uno de los objetivos principales del diseño sísmico, existe incertidumbre en los procedimientos actuales para proporcionar un margen de seguridad adecuado contra la inestabilidad dinámica. El efecto desestabilizador de las cargas gravitacionales en edificios sujetos a movimientos severos del terreno puede llevarlos al colapso cuando son forzados a desarrollar incursiones inelásticas importantes durante la respuesta. Este efecto se manifiesta sobre la estructura lateralmente deformada incrementando los momentos en la base de los elementos verticales (efecto P- $\Delta$ ), lo que conduce a que durante la ocurrencia del sismo se incremente cada vez más la respuesta, conduciendo a la estructura a la inestabilidad dinámica. Dado lo anterior, vale la pena mencionar como ejemplo que los niveles de desempeño estructural definidos por el FEMA 350 se asocian a valores predefinidos de parámetros de respuesta (distorsión de entrepiso entre otros) y que específicamente para el estado límite Colapso Global la inestabilidad dinámica en la estructura es ya notoria (Vamvatsikos y Cornell, (2002)).

Estudios sobre la inestabilidad dinámica de estructuras han señalado el papel que el mecanismo plástico juega en la seguridad de la estructura contra colapso (Nakajima et al., 1990; Bernal, 1992 y 1998; Sordo y Bernal, 1993). Estos estudios han reportado que diseños sísmicos comunes no aseguran necesariamente un nivel adecuado de seguridad contra la inestabilidad, dependiendo dicho nivel de la sobreresistencia y del mecanismo de falla que ocurre. Estos trabajos han caracterizado el problema a partir de las propiedades elásticas de la estructura, la resistencia última y la forma del mecanismo de colapso.

Bernal (1992) ha propuesto una metodología para estructuras planas basada en la reducción de la estructura a un sistema de un grado de libertad equivalente, obtenido a través de un procedimiento de empujón. Dado que la geometría tridimensional de la mayoría de los edificios reales introduce la posibilidad de desarrollar mecanismos de falla rotacionales (Sordo y Bernal, 1994 y 1996) y que el efecto de bidireccionalidad de los movimientos del terreno y el ángulo de incidencia de éste sobre la estructura agrega complejidad al problema, se ha propuesto (Sordo y Bernal, 1994; Sordo y Félix, 2000) un enfoque atractivo para simplificar el problema a través de la definición de un sistema de un grado de libertad con propiedades de disipación de energía equivalentes a las de la estructura tridimensional.

Por otro lado, Zarfam y Mofid (2011) han estudiado la conveniencia de adaptar la contribución de los modos superiores al análisis dinámico incremental (IDA), mediante la idealización de la curva de capacidad como una curva trilineal, la cual es asignada a los sistemas de un grado de libertad trilineales para representar la respuesta de las estructuras en sus modos superiores. El trabajo mencionado utiliza el modelo trilineal para representar estructuras propiamente de concreto reforzado, aunque se ha visto que el modelo es aplicable también a estructuras de acero con niveles considerables de carga gravitacional (Díaz, 2005) para representar la transición entre pendiente positiva y negativa de la curva de capacidad.

En los casos indicados antes, los sistemas de un grado de libertad (SUGL) se formulan a través de las propiedades elásticas de la estructura, su resistencia última (en términos energéticos o de fuerza cortante) y la forma del mecanismo plástico. Sin embargo, no se han hecho a la fecha estudios detallados sobre las variaciones que las estimaciones de la seguridad contra inestabilidad dinámica puedan presentar debido a las características mismas del proceso de formación del mecanismo. Mientras Bernal (1992) asegura que estas características no afectan la estimación del coeficiente de colapso por inestabilidad dinámica, otros estudios indican que este factor sufre modificaciones cuando la transición elastoplástica de la estructura varía de un caso a otro (Sordo y Díaz, 2003). En este trabajo se pretende ofrecer un paso más en esta dirección, estimando las variaciones en el coeficiente de colapso $(C c)$ por inestabilidad dinámica que se pueden presentar debido a las características del proceso de formación del mecanismo plástico dado que las características elásticas, el cortante resistente y la forma del mecanismo se mantienen constantes. 
En la primera parte del trabajo se estudia el comportamiento de un conjunto de marcos planos (marcos de referencia), de los cuales se deriva a su vez otro conjunto de marcos (submodelos) con los que se ilustran las importantes diferencias que se pueden llegar a presentar en el coeficiente de colapso por inestabilidad dinámica debido a las diferencias en la formación del mecanismo de colapso (Sordo y Díaz, 2003; Díaz y Sordo, 2004). Dado el carácter teórico del estudio, los submodelos se generan a partir de los marcos de referencia "artificialmente" modificando las resistencias de los elementos estructurales que conforman las estructuras (como se explica más adelante). Tales marcos se someten a la acción de un grupo de registros acelerográficos para determinar la influencia de las características de los mismos. Con este análisis se determinan tendencias cualitativas relacionadas con las diferentes geometrías estructurales propuestas, sus mecanismos plásticos y las características propias de los registros acelerométricos.

En la segunda parte del estudio se analizan sistemas de un grado de libertad con ciclo histerético trilineal (SUGLT) para evaluar los parámetros que pueden resultar importantes para caracterizar el efecto de la transición elastoplástica en el comportamiento de estructuras en el umbral de la inestabilidad dinámica. El uso de sistemas trilineales obedece a la necesidad de considerar de una manera más detallada (en comparación con sistemas elastoplásticos) la transición elastoplástica en el presente trabajo.

De los resultados del trabajo se observan diferencias de hasta un $50 \%$ en el coeficiente de colapso entre sistemas con las mismas propiedades elásticas, cortante resistente y mecanismo plástico. La influencia de la transición del estado elástico al plástico en los valores que toma el coeficiente de colapso por inestabilidad dinámica es más acentuada al utilizar registros con epicentro lejano. Los sistemas elastoplásticos muestran tener un $C c$ más alto que los sistemas con transición elastoplástica paulatina.

\section{CONSIDERACIONES Y DEFINICIONES PRELIMINARES}

Durante el desarrollo del trabajo se hacen las consideraciones que se mencionan a continuación.

- En los análisis de marcos planos se considera que la inestabilidad dinámica (colapso dinámico) se presenta cuando el desplazamiento máximo de azotea en un análisis considerando efecto P- $\Delta$ es cuatro veces tal desplazamiento cuando éste se ignora (Díaz y Sordo, 2004).

- En el análisis de sistemas de un grado de libertad con ciclo histerético trilineal (SUGLT), la inestabilidad dinámica se presenta, cuando durante la respuesta se alcanza cierto nivel de desplazamiento (desplazamiento de colapso) definido más adelante.

- El coeficiente de colapso por inestabilidad dinámica resulta ser el coeficiente sísmico dividido por el factor de comportamiento sísmico y por el valor por el cual se escalan las aceleraciones de un registro para que induzca comportamiento inestable en un sistema (estructural o de un grado de libertad) en un análisis dinámico.

- En los análisis dinámicos, el escalamiento de los registros se hace multiplicando la historia de aceleraciones en cuestión por un número real mayor que cero.

- Se considera que los elementos de cada estructura cuentan con capacidad suficiente (por ejemplo capacidad de rotación) para lograr la formación del mecanismo plástico, es decir, el único estado límite revisado en el presente trabajo es la inestabilidad dinámica.

- Durante los análisis dinámicos no se considera el efecto que la velocidad de carga tiene sobre la resistencia del material ni endurecimiento por deformación del acero. 


\section{MARCOS PLANOS}

Como primer paso se estudia la influencia que las características de la transición del estado elástico al plástico en marcos planos de acero pueden llegar a tener en el coeficiente de colapso. Para las estructuras estudiadas se asegura que se generen mecanismos plásticos específicos predefinidos. Para cada geometría estructural (marco de referencia) se definen submodelos que conservan sus características elásticas como: rigidez inicial, periodo fundamental, mecanismo de colapso y cortante basal último (obtenidos mediante un proceso de empujón estático con un patrón de cargas proporcional a las masas, modificando resistencias de elementos que conforman el mecanismo de colapso y no haciendo nuevos diseños); siendo la única diferencia entre submodelos la transición del estado elástico a la formación del mecanismo de falla.

\section{Marcos de Referencia}

Para la primera parte del trabajo se consideraron cinco marcos planos de acero resultado de un prediseño por resistencia para un coeficiente sísmico $c=0.40$, para los cuales se buscan diferentes configuraciones de su mecanismo de colapso y que a final de cuentas dado el problema a estudiar es necesario revisar diferentes mecanismos de colapso para cada marco de referencia (Díaz, 2005), esto en el entendido que dichos mecanismos (no globales) resultan insatisfactorios en la práctica de la ingeniería. Tales marcos se someten a análisis dinámicos no lineales hasta llevarlos al umbral de la inestabilidad dinámica. Para la ejecución de los análisis dinámicos se considera un amortiguamiento del $2 \%$ del crítico y un comportamiento elastoplástico perfecto. Los efectos P- $\Delta$ actuando sobre cada estructura se evalúan mediante la formulación de la matriz de rigidez geométrica (Tena, 2007).

La geometría de los marcos de referencia junto con un esquema de su mecanismo de colapso y el periodo del primer modo de vibración $(T)$ se muestra en la figura 1, asimismo se muestra el valor del coeficiente de estabilidad asociado a cada uno de los modelos $(\Theta)$. Para el caso de estructuras bidimensionales y conociendo de antemano el mecanismo de falla que ocurre, este coeficiente se calcula de acuerdo a las siguientes expresiones (Bernal, 1992).

$\Theta=\frac{\theta_{m}}{Q_{0}}$

en la ecuación anterior $\Theta$ es el coeficiente de estabilidad de la estructura, $\theta_{m}$ es un parámetro adimensional, se calcula como:

$\theta_{m}=\frac{\tau g \Omega_{m}}{\omega_{0}^{2} h}$

siendo $\tau$ la razón de la carga total gravitacional a la carga muerta, $g$ la aceleración de la gravedad, $\omega_{0}$ es la frecuencia natural cíclica del primer modo de vibración de la estructura, $h$ la altura total del sistema estructural y $\Omega_{m}$ es un parámetro adimensional, que relaciona la forma del mecanismo plástico con la altura de la estructura, se calcula como: 
$\Omega_{m}=\frac{\left(1-\frac{G}{2 h}-\frac{E}{h}\right)}{\frac{G}{h}\left(1-\frac{2 G}{3 h}-\frac{E}{h}\right)}$

$E$ y $G$ están en función de la forma del mecanismo plástico y se definen en la figura 1 . $E$ es la altura que hay de la base y hasta el primer nivel involucrado en el mecanismo de colapso, en los primeros cuatro marcos mencionados en la figura $1 E=0, G$ es la altura total de los niveles involucrados en el mecanismo de colapso, por ejemplo para el marco JAC05 $G=15.0 \mathrm{~m}$.

$Q_{0} \quad$ parámetro adimensional calculado con la siguiente expresión

$Q_{0}=1-\theta_{0}+\theta_{m}$

donde $\Omega_{m}$ es un parámetro adimensional que relaciona la forma del mecanismo plástico con la altura de la estructura, se calcula como:

$\theta_{0}=\frac{\tau g \Omega_{0}}{\omega_{0}^{2} h}$

$\tau$, $g, \omega_{0}, h$ han sido definidos antes, $\Omega_{0}$ es un parámetro que considera el número de niveles de la estructura, se calcula como:

$\Omega_{0}=\frac{3 n}{2 n+1}$

$n$ es el número de niveles de la estructura.

El coeficiente de estabilidad es una medida cuantitativa del efecto desestabilizante de las cargas gravitacionales sobre la estructura, toma valores entre cero y uno. Por ejemplo $\Theta=0$ implica que no existen efectos P- $\Delta$ actuando en la estructura. $\Theta=1.0$ Implica inestabilidad estática en la estructura.

De la figura 1 se observa que los marcos de referencia JAC05 y JAK05 poseen la misma geometría y forma de mecanismo. Los elementos estructurales que los conforman son los mismos, siendo la única diferencia el nivel de carga gravitacional que actúa en las columnas de cada marco. Para el marco de referencia JAK05 la carga gravitacional transmitida a las columnas por sistemas perpendiculares es cuatro veces mayor que en el caso del marco JAC05. Por otro lado, en el caso de los marcos de referencia OBE04 y OBE23, éstos comparten únicamente la geometría general puesto que los elementos que los componen son diferentes y como se puede ver en la figura 1, la forma del mecanismo de falla de ambos marcos también es distinta.

\section{Obtención de los submodelos para cada Marco de Referencia}

Cada marco de referencia se somete a un procedimiento de empujón estático con carga proporcional a las masas de cada entrepiso (programa de análisis estructural DRAIN 2DX, Prakash et al., 1993) y se obtiene la curva cortante basal contra desplazamiento de azotea como se muestra en la figura 2. 


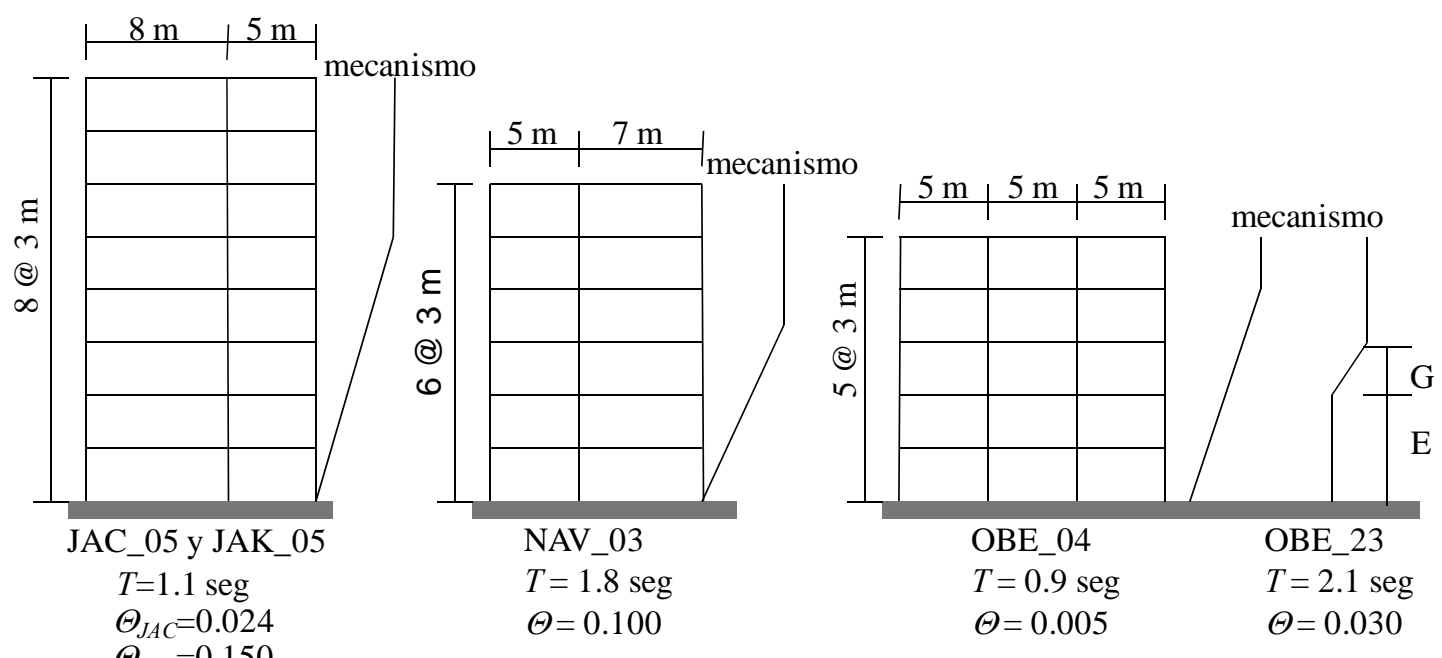

Figura 1. Vista en elevación de los marcos estudiados

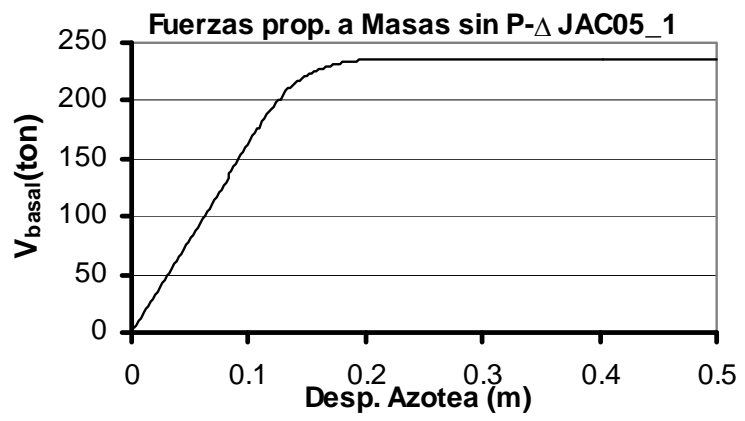

Figura 2. Cortante basal vs. desplazamiento de azotea para JAC05_1

De los resultados arrojados por el pushover con cargas proporcionales a las masas se determina el mecanismo de falla que se presenta en cada marco de referencia, mismo que ha de conservarse para los submodelos de cada uno de éstos. Por ejemplo, en la figura 3 se puede ver el mecanismo asociado a los diferentes marcos de referencia. Los círculos de la figura indican las articulaciones plásticas que aparecen en la estructura y que definen el mecanismo de falla.

A partir de cada marco de referencia se generan cuatro submodelos para los que se induce un mecanismo específico (que es el mismo que el del marco de referencia) con diferentes secuencias de formación de articulaciones plásticas. Lo anterior se consigue modificando resistencias de elementos predefinidos, es decir, a las secciones que no intervienen en el mecanismo plástico se les asignan resistencias muy grandes para garantizar que el mecanismo no variará entre submodelos y a los elementos que se articulan para formar el mecanismo se les hace variar su resistencia de tal forma que el orden en que aparecen las articulaciones es aleatorio y distinto entre los diferentes submodelos, esto último sin variar la capacidad de cortante basal de la estructura. Así se obtienen los diferentes submodelos que van desde aquel que presenta una pérdida de rigidez repentina (modelos aproximadamente bilineales con ductilidades y sobreresistencias cercanas a 1.0), hasta aquellos que presentan una transición paulatina entre el estado elástico y la formación del mecanismo plástico. El procedimiento anterior se verifica mediante análisis de empujón estático para asegurar que se genera el mismo mecanismo del marco de referencia en los cuatro marcos derivados de éste. Las características de los elementos que conforman cada estructura se muestran en detalle en Díaz (2005). 


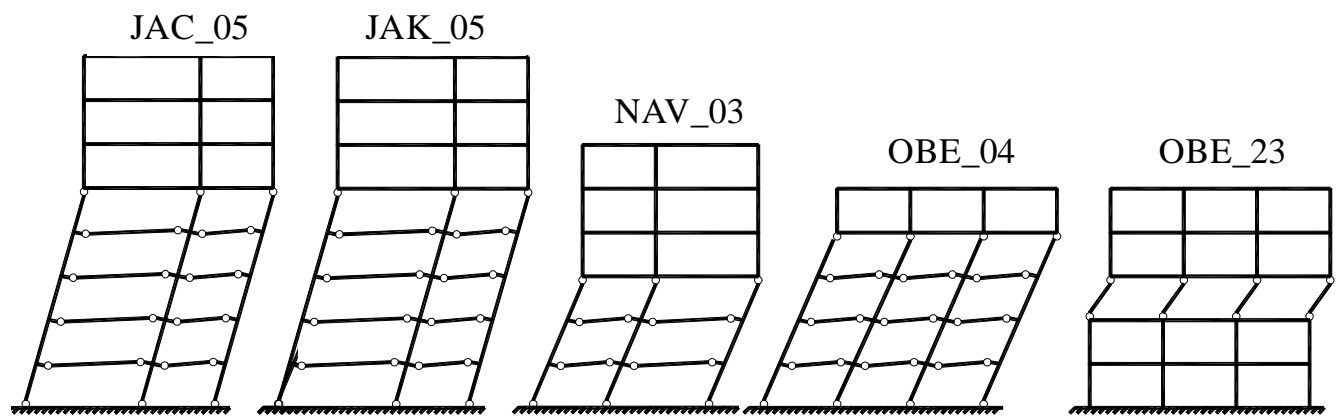

Figura 3. Mecanismo plástico de los marcos de referencia

Lo anterior se hace con la finalidad de estudiar las diferencias que pudieran existir en el coeficiente de colapso dinámico, al considerar modelos bilineales o multilineales (transición paulatina) con los que se pudiera representar de manera más aproximada el comportamiento de transición elastoplástica real. Para comparar, se aplican empujones con patrones de carga incremental proporcional a las masas hasta la formación del mecanismo, observándose las diferencias que se muestran en la figura 4 sin considerar efectos P- $\Delta$ y en la figura 5 considerando efectos P- $\Delta$. Estas diferencias entre los submodelos de un mismo marco de referencia son las que se estudian en el presente trabajo para determinar si influyen en el coeficiente de colapso por inestabilidad dinámica.
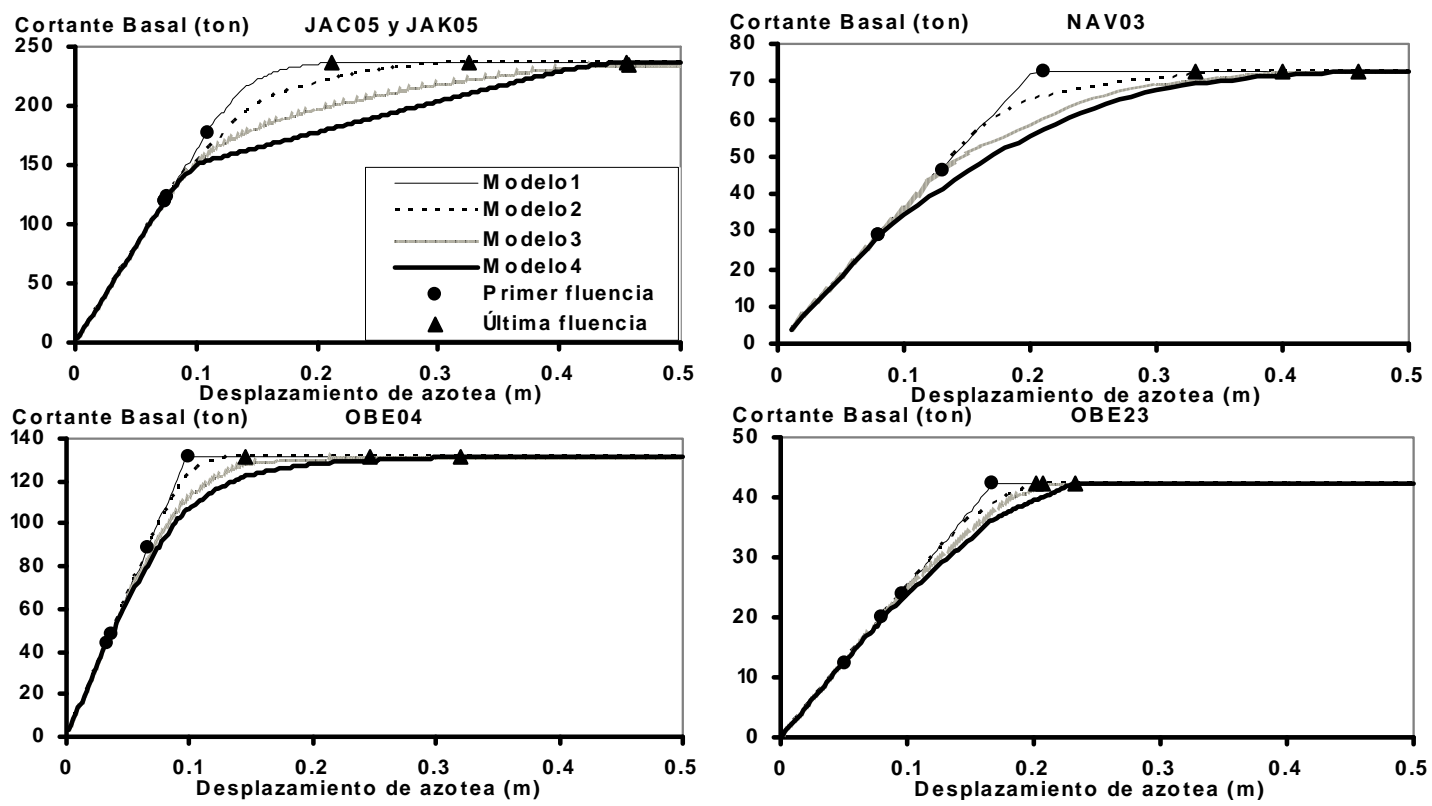

Figura 4. Cortante basal $v s$. desplazamiento de azotea para los marcos de referencia sin efecto P- $\Delta$

En las figuras 4 y 5 se puede observar la influencia negativa que sobre la estabilidad de la estructura tiene el efecto P- $\Delta$ cuando se genera un mecanismo local o de entrepiso suave, en particular, las curvas del marco de referencia OBE23 muestran una pérdida drástica de la rigidez de la estructura precisamente por la generación de dicho mecanismo. La tabla 1 muestra parámetros asociados a la curva de capacidad de los submodelos en estudio. 
Gerardo Díaz Martínez y Emilio Sordo Zabay

Tabla 1. Parámetros asociados a la formación del mecanismo en los marcos en estudio

\begin{tabular}{|c|c|c|c|c|c|c|c|}
\hline $\begin{array}{l}\text { Marco de } \\
\text { referencia } \\
\end{array}$ & Submodelo & $\begin{array}{c}\text { Cortante de } \\
\text { Fluencia } V_{y} \text { (ton) } \\
\end{array}$ & $\begin{array}{c}\text { Desplazamiento de } \\
\text { fluencia } \Delta_{y}(\mathrm{~m})\end{array}$ & $\begin{array}{c}\text { Cortante último } \\
V_{u} \text { (ton) }\end{array}$ & $\begin{array}{c}\text { Desplazamiento } \\
\text { último } \Delta_{u}(\mathrm{~m})\end{array}$ & $\begin{array}{c}\text { Sobreresistencia } \\
\left(V_{u} / V_{y}\right)\end{array}$ & $\begin{array}{c}\text { Ductilidad } \\
\left(\Delta_{u} / \Delta_{v}\right) \\
\end{array}$ \\
\hline \multirow{4}{*}{ JAC05 } & 1 & 176.23 & 0.110 & 236.44 & 0.212 & 1.34 & 1.927 \\
\hline & 2 & 119.60 & 0.074 & 236.44 & 0.326 & 1.98 & 4.405 \\
\hline & 3 & 118.60 & 0.074 & 236.44 & 0.458 & 1.99 & 6.189 \\
\hline & 4 & 122.31 & 0.076 & 236.44 & 0.456 & 1.93 & 6.000 \\
\hline \multirow{4}{*}{ JAK05 } & 1 & 169.75 & 0.106 & 236.44 & 0.222 & 1.39 & 2.094 \\
\hline & 2 & 120.71 & 0.076 & 236.44 & 0.336 & 1.96 & 4.421 \\
\hline & 3 & 120.71 & 0.076 & 236.44 & 0.470 & 1.96 & 6.184 \\
\hline & 4 & 112.24 & 0.070 & 236.44 & 0.484 & 2.11 & 6.914 \\
\hline \multirow{4}{*}{ NAV03 } & 1 & 72.63 & 0.21 & 72.63 & 0.210 & 1.00 & 1.000 \\
\hline & 2 & 28.78 & 0.08 & 72.63 & 0.330 & 2.52 & 4.125 \\
\hline & 3 & 46.39 & 0.130 & 72.63 & 0.400 & 1.57 & 3.077 \\
\hline & 4 & 28.70 & 0.080 & 72.63 & 0.460 & 2.53 & 5.750 \\
\hline \multirow{4}{*}{ OBE04 } & 1 & 131.34 & 0.100 & 131.34 & 0.100 & 1.00 & 1.00 \\
\hline & 2 & 88.26 & 0.066 & 131.40 & 0.146 & 1.49 & 2.212 \\
\hline & 3 & 48.40 & 0.036 & 131.41 & 0.246 & 2.72 & 6.833 \\
\hline & 4 & 43.47 & 0.034 & 131.41 & 0.320 & 3.02 & 9.41 \\
\hline \multirow{4}{*}{ OBE23 } & 1 & 42.20 & 0.168 & 42.204 & 0.168 & 1.00 & 1.000 \\
\hline & 2 & 23.77 & 0.096 & 42.204 & 0.204 & 1.78 & 2.125 \\
\hline & 3 & 19.98 & 0.080 & 42.204 & 0.208 & 2.11 & 2.600 \\
\hline & 4 & 12.38 & 0.050 & 42.204 & 0.232 & 3.41 & 4.640 \\
\hline
\end{tabular}

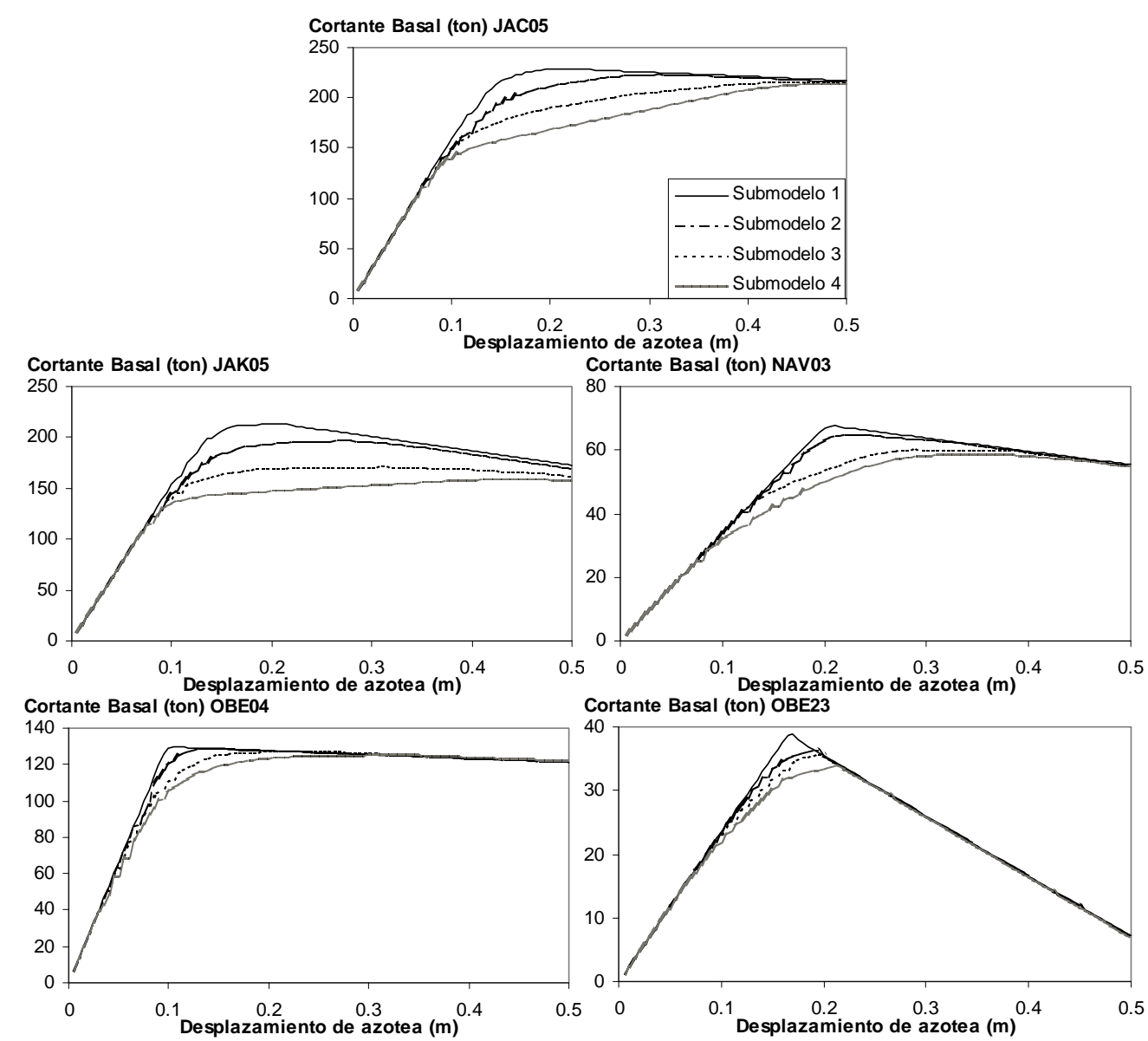

Figura 5. Curvas de capacidad para los marcos de referencia con efecto P- $\Delta$ 
En la figura 6 se muestra gráficamente el significado de los parámetros mostrados en la tabla 1. Para la generación de los submodelos de cada marco de referencia se procura que la ductilidad y en su caso la sobreresistencia crezcan entre los mismos y que el cortante basal último sea el mismo para cada marco y sus submodelos. Este último hecho es el que define la forma de las curvas de capacidad de cada submodelo puesto que al aumentar la sobreresistencia y la ductilidad de la estructura la pérdida de rigidez es más gradual y de ahí las diferencias en las curvas entre submodelos de un mismo marco de referencia (figura 4).

\section{Obtención de las curvas de segundo ciclo de empujón histerético}

Dado que un estudio previo (Sordo y Díaz 2003) ha llegado a la conclusión de que es conveniente considerar la forma de la curva cortante-desplazamiento en ciclos posteriores al primer empujón para efectos de reducción a sistemas de un grado de libertad para calcular el coeficiente de colapso dinámico, en especial si los efectos desestabilizantes de la carga gravitacional son importantes; se explora en el siguiente trabajo la posibilidad de que la forma del segundo ciclo de las curvas de capacidad de los marcos en estudio pueda predecir posibles diferencias en el coeficiente de colapso entre submodelos de un mismo marco de referencia. Lo anterior se basa en el hecho de que las curvas de ciclos posteriores al primer empujón exhiben diferencias más marcadas entre submodelos de un marco de referencia. En la figura 7 se puede ver la forma de una curva cortante basal vs. desplazamiento de azotea para una estructura sometida a un proceso de empujón estático histerético. En la figura 7 se muestran también los puntos y las líneas auxiliares que se utilizan para la generación de las curvas de segundo ciclo de empujón estático.

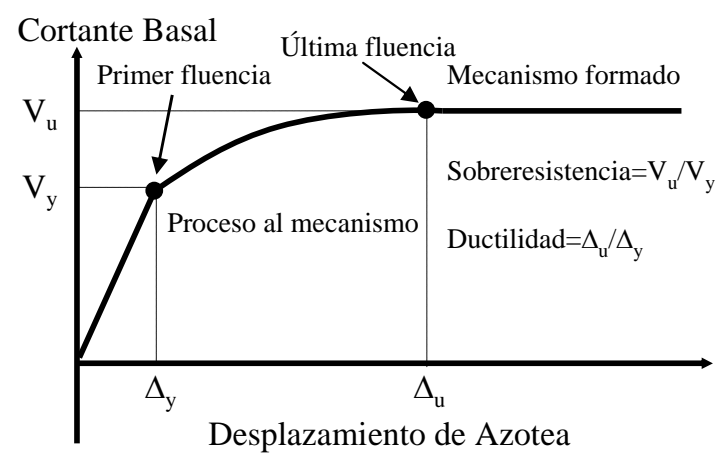

Figura 6. Parámetros asociados a la formación del mecanismo de los marcos en estudio

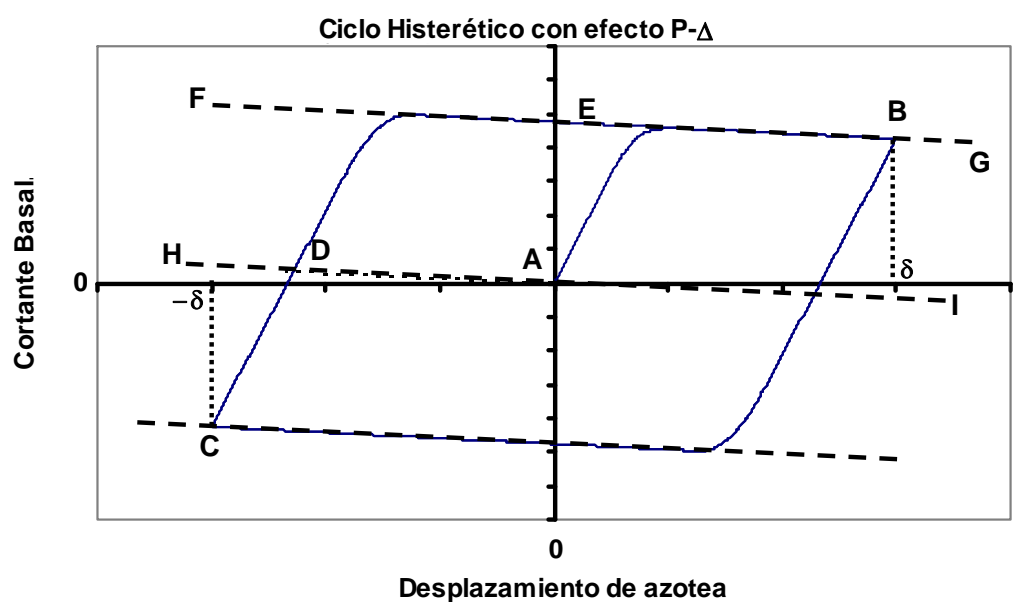

Figura 7. Curva cortante basal vs. desplazamiento de azotea para una estructura sometida a proceso de empujón cíclico 
En la figura 7, la curva que va del punto A al punto B (el punto B de descarga se ha elegido de tal forma que el mecanismo plástico se ha formado) denota el comportamiento de la estructura cuando se somete a un proceso de empujón estático hasta determinado nivel de desplazamiento de azotea $(\delta)$. Si a partir del punto B se impone un desplazamiento opuesto a la estructura de $-2 \delta$ hasta que llega al punto $\mathrm{C}$ y a partir de $C$ se impone nuevamente un desplazamiento de azotea a la estructura de $2 \delta$ hasta el punto B se obtiene una curva cuya transición elastoplástica es en muchos casos diferente a la monotónica original.

Dada la complejidad del comportamiento histerético cíclico no lineal de las estructuras, por simplicidad se estudiarán en el presente trabajo las curvas monotónicas (primer ciclo) y la correspondiente al segundo ciclo de histéresis de la curva, obtenida ésta de la siguiente metodología, la cual de forma cualitativa trata de considerar la pendiente negativa de la curva histerética una vez que el mecanismo plástico se ha generado (ver figura 7):

- $\quad$ En primer lugar se traza la línea auxiliar FG la cual es paralela a la recta de pendiente negativa de la curva $A B$ que se genera después que el mecanismo de falla de la estructura se ha formado.

- Se traza una paralela a la recta FG (HI) que pase por el origen del sistema de ejes coordenados.

- El punto D donde la recta HI corta a la curva definida por los puntos C y B será el origen de la curva cortante basal contra desplazamiento para el segundo ciclo de histéresis. El punto final (punto E de la figura 7) está determinado por el nivel de desplazamiento deseado.

El punto D (origen de las curvas de segundo ciclo de empujón estático) se genera de la forma mencionada para evitar, por la forma de la hitéresis de los modelos, que se presente en ciclos posteriores cortante basal último mayor que el cortante basal último del primer ciclo. De lo anterior, la figura 8 muestra las curvas de segundo ciclo de empujón para los modelos estudiados, en todos los casos el origen del sistema de ejes se ha hecho coincidir con el punto que correspondería al punto D de la figura 7.

Se puede ver de la comparación de las figuras 5 y 8 que las diferencias en la transición del estado elástico al plástico son más notorias en las curvas del segundo ciclo de empujón estático, más adelante, la importancia de dicha transición en las variaciones del coeficiente de colapso por inestabilidad dinámica trata de explicarse en función de la forma de estas curvas.

\section{Trabajo Monotónico Máximo}

Se obtiene el trabajo requerido para llevar a la estructura a un nivel de desplazamientos tal que el cortante actuante asociado sea cero y el patrón de desplazamientos se encuentre en equilibrio inestable con la carga gravitacional $(\mathrm{Wm})$. Este parámetro se propone en algunos estudios como un posible indicador de la seguridad contra colapso dinámico en sistemas de varios grados de libertad (Sordo y Félix; 2000, Sordo y Bernal, 1994). La figura 9 muestra una curva de capacidad típica para la cual se obtiene el trabajo realizado por el sistema para llegar a la condición de cortante basal igual a cero.

El cálculo del trabajo se hace para todos los submodelos del estudio sometidos a procedimiento de empujón estático ante un patrón de cargas proporcional a las masas de entrepiso, la finalidad es determinar si el trabajo estático es un parámetro que pueda predecir en qué casos la transición del estado elástico al plástico en estructuras influye en el coeficiente de colapso dinámico. La figura 10 muestra una gráfica del trabajo monotónico contra el desplazamiento de azotea. 
Efecto de la transición al mecanismo plástico en la inestabilidad dinámica
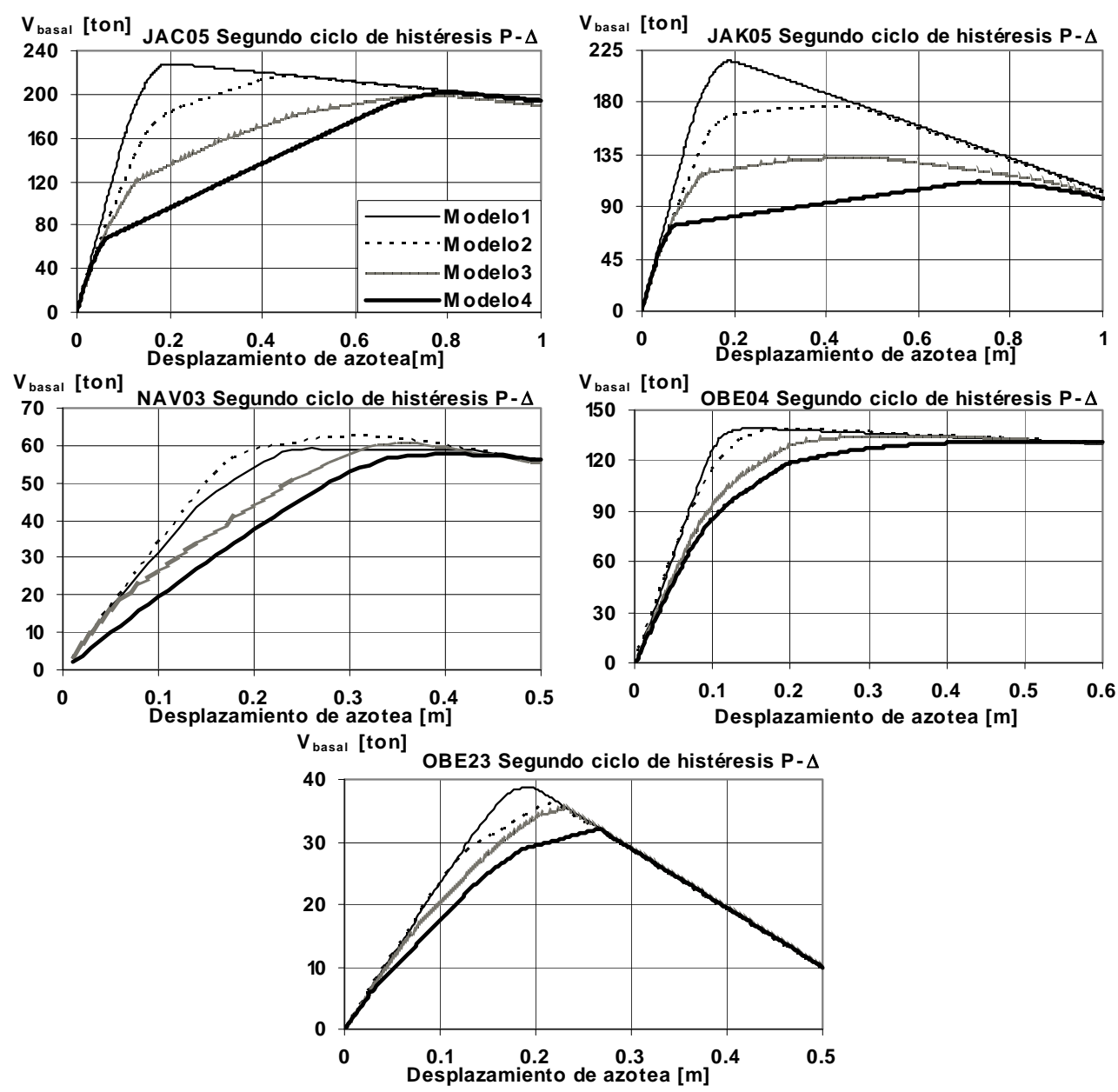

Figura 8. Curvas de capacidad de los modelos para el segundo ciclo histerético de empujón

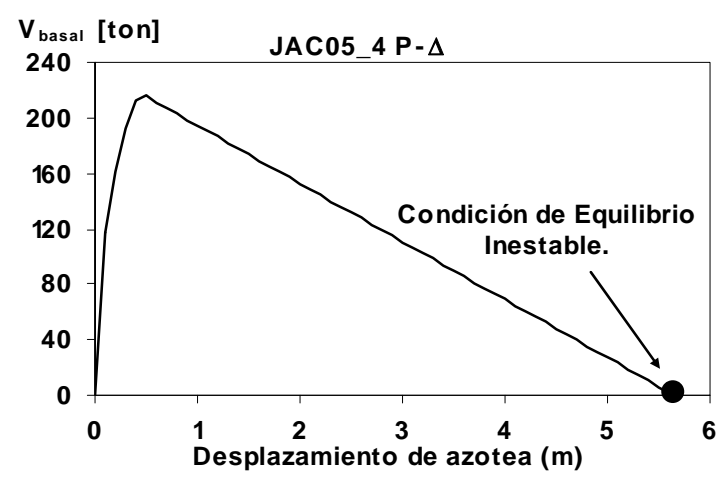

Figura 9. Curva de capacidad submodelo JAC05_4

El máximo en la curva de la figura 10 corresponde al trabajo realizado hasta el punto en el que el cortante basal vale cero en la curva cortante basal versus desplazamiento de azotea, definido como $\mathrm{Wm}$ para cada submodelo. En la tabla 2 se muestran los valores de $W m$ para todos los modelos en estudio para el primer ciclo de empujón estático. La tabla 3 muestra los valores de $\mathrm{Wm}$ para todos los modelos en estudio para el segundo ciclo de empujón estático. 


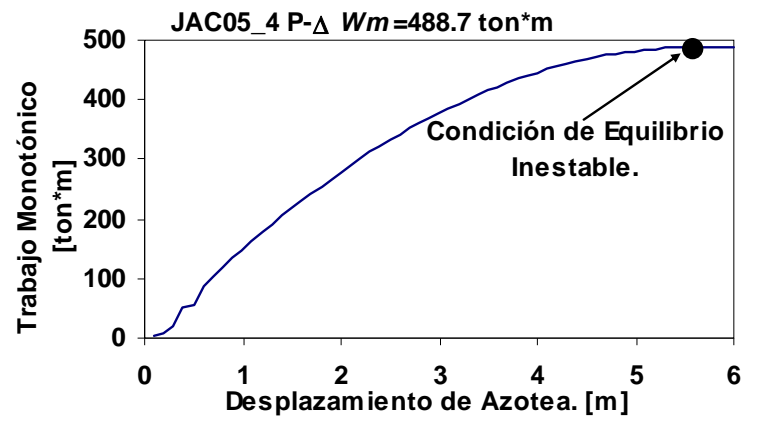

Figura 10. Trabajo bajo carga monotónica para el submodelo JAC05_4

De la tabla 2 se puede observar que no existen diferencias importantes de los resultados reportados del trabajo monotónico máximo para primer ciclo de empujón estático entre los modelos en estudio, se puede concluir preliminarmente que el trabajo monotónico máximo correspondiente al primer ciclo de empujón estático no es un parámetro indicativo de los casos en que la transición elastoplástica influye sobre el $C c$.

En la tabla 3 se aprecian diferencias de importancia del trabajo monotónico máximo entre submodelos de un mismo marco de referencia especialmente en el caso de JAK05, en general el segundo ciclo muestra mayores diferencias en dicho trabajo, pareciera que tales diferencias pueden predecir en qué casos es influyente la transición del estado elástico al plástico en el $C c$ como se verá más adelante.

Tabla 2. Trabajo monotónico máximo ( $\left.\mathrm{Wm}\left[\mathrm{ton}^{*} \mathrm{~m}\right]\right)$ para el primer ciclo de empujón estático

\begin{tabular}{cccccc}
\hline \multicolumn{5}{c}{ Primer ciclo } \\
\hline Modelo & JAC05 & JAK05 & NAV03 & OBE04 & OBE23 \\
1 & 493.2 & 157.4 & 52.7 & 196.8 & 3.7 \\
2 & 495.2 & 159.7 & 52.4 & 197.1 & 3.7 \\
3 & 495.5 & 158.7 & 51.4 & 197.5 & 3.7 \\
4 & 488.7 & 158.9 & 49.9 & 196.2 & 3.6 \\
Max/min & 1.014 & 1.015 & 1.056 & 1.007 & 1.042 \\
\hline \multicolumn{7}{c}{}
\end{tabular}

Tabla 3. Trabajo monotónico máximo ( $W m$ [ton*m]) para el segundo ciclo de empujón estático

\begin{tabular}{cccccc}
\hline \multicolumn{5}{c}{ Segundo ciclo } \\
\hline Modelo & JAC05 & JAK05 & NAV03 & OBE04 & OBE23 \\
1 & 494.4 & 144.1 & 50.1 & 196.6 & 4.9 \\
2 & 484.1 & 130.5 & 50.9 & 197.0 & 4.8 \\
3 & 465.1 & 155.3 & 48.3 & 195.4 & 4.7 \\
4 & 445.1 & 65.9 & 45.3 & 192.3 & 4.5 \\
Max/min & 1.111 & 2.357 & 1.124 & 1.024 & 1.096 \\
\hline
\end{tabular}

\section{Registros sísmicos utilizados en el estudio}

Los marcos definidos anteriormente se someten a la acción de veinte registros de sismos reales (ver tabla 4); 16 de los cuales son registros recomendados y clasificados por el Pacific Earthquake Engineering Research Center, (Elgamal et al., 1998). Para información más detallada acerca de los registros utilizados en el estudio ver Díaz (2005). 
Efecto de la transición al mecanismo plástico en la inestabilidad dinámica

Tabla 4. Registros utilizados en el trabajo

\begin{tabular}{|c|c|c|c|c|c|c|}
\hline Registro & Epicentro & Tipo de Suelo & $\begin{array}{l}\text { Aceleración } \\
\text { máxima (g) }\end{array}$ & $\begin{array}{c}\text { Velocidad } \\
\text { máxima }\left(\mathrm{m} / \mathrm{s}^{2}\right)\end{array}$ & Clasificación & Sitio \\
\hline CAP000 & Cercano & Firme & 1.497 & 1.310 & CR & Cape Mendocino \\
\hline КОВ000 & Cercano & Firme & 0.834 & 0.921 & CR & Kobe JMA \\
\hline LGP000 & Cercano & Firme & 0.510 & 0.962 & CR & Loma Prieta, LGPC \\
\hline LUC000 & Cercano & Firme & 0.671 & 0.359 & CR & Landers, Lucerna \\
\hline MEL000 & Cercano & Blando & 0.316 & 0.697 & CS & Imperial Valley, Meloland \\
\hline NRR228 & Cercano & Blando & 0.826 & 1.740 & CS & Northridge, Rinaldi Receiving st. \\
\hline PET000 & Cercano & Blando & 0.589 & 0.464 & CS & Cape Mendocino, Petrolia \\
\hline TAB074 & Cercano & Blando & 0.880 & 0.983 & CS & Tabas Irán, Tabas \\
\hline CAL000 & Lejano & Firme & 0.141 & 0.166 & LR & Michoacán, Caleta de Campos \\
\hline OCT180 & Lejano & Firme & 0.049 & 0.070 & LR & Michoacán \\
\hline OFU041 & Lejano & Firme & 0.211 & 0.130 & LR & Miyagi-oki, Ofunato \\
\hline VAL070 & Lejano & Firme & 0.168 & 0.150 & LR & Chile Valu \\
\hline LLO010 & Lejano & Blando & 0.658 & 0.414 & LS & Chile. Llollelo \\
\hline SAB182 & Lejano & Blando & 0.068 & 0.079 & LS & Olimpia, Seattle Army Base \\
\hline TAC180 & Lejano & Blando & 0.035 & 0.114 & LS & Michoacán \\
\hline TOK000 & Lejano & Blando & 0.318 & 0.427 & LS & Tokachi-oki, Hachinohe Harbor \\
\hline CEN000 & Lejano & Firme & 0.349 & 0.381 & LR & El Centro, California \\
\hline TAF021 & Lejano & Firme & 0.156 & 0.182 & LR & Kern County \\
\hline SCT090 & Lejano & Blando & 0.168 & 0.595 & LS & Ciudad de México \\
\hline CDA000 & Lejano & Blando & 0.082 & 0.417 & LS & Central de Abastos, México D.F. \\
\hline
\end{tabular}

Adicionalmente a los 16 primeros registros de la tabla 4, se han utilizado cuatro más (cuatro últimos registros en la tabla 4) y se hace una clasificación que trata de ser acorde a los grupos mencionados.

\section{Cálculo del Coeficiente de Colapso Dinámico}

Para cada registro se obtiene la respuesta de cada modelo para diferentes valores del coeficiente sísmico (utilizado en el prediseño) desde un valor inicial que induce comportamiento elástico hasta un valor para el cual en la estructura el colapso es inminente.

Se define el coeficiente de colapso dinámico $(C c)$, como el valor mínimo del coeficiente sísmico de cortante basal requerido para prevenir la inestabilidad dinámica, en forma aproximada el coeficiente se obtiene a través del factor de escala de las aceleraciones del registro (también conocido como factor de seguridad contra inestabilidad dinámica Bernal, 1992) que induce comportamiento inestable en la estructura, como lo muestra la ecuación 7

$C c=\left(\frac{c}{Q}\right) \frac{1}{I_{c}}$

donde

$\frac{c}{Q} \quad$ coeficiente de cortante basal de diseño en este estudio se toma igual a 0.20

$I_{c} \quad$ Factor de escala de las aceleraciones del registro que induce inestabilidad dinámica.

El hecho de amplificar las aceleraciones en los registros tiene por objeto como se mencionó anteriormente obtener en forma aproximada el $C$. Estudios recientes han encontrado que no existe evidencia estadística que indique que se incurre en errores importantes al escalar registros acelerométricos únicamente multiplicando la historia de aceleraciones por un número real mayor que cero; cuando se requiere calcular parámetros de respuesta no lineal (Díaz y Sordo, 2008; Iervolino y Cornell, 2005). 
En la figura 11 se muestra una curva desplazamiento de azotea máximo contra coeficiente sísmico de cortante basal de diseño $(c /(Q I))$, dicho coeficiente se reduce de manera aproximada escalando las aceleraciones del registro en cuestión, así para un factor de aceleraciones de 1.0 el coeficiente basal toma el valor de 0.2. Para la obtención de la curva de la figura 11 se hace el análisis dinámico de la estructura (en este caso se utiliza el registro SCT090) y se toma el valor del desplazamiento de azotea máximo, siendo éste, junto con $c$ el primer punto de la curva. Para la obtención del siguiente punto de la curva se reduce el coeficiente sísmico (amplificando las aceleraciones del registro) y se vuelve a obtener el desplazamiento máximo de azotea de la estructura durante su respuesta, el proceso continúa hasta que el desplazamiento máximo de azotea considerando efectos P- $\Delta$ es cuatro veces el desplazamiento máximo sin considerar tales efectos ( $\alpha=4$ como se verá más adelante), este último valor del coeficiente sísmico es el coeficiente de colapso dinámico $(C c)$. La figura 11 muestra cualitativamente la obtención del coeficiente de colapso $(C c)$ en un caso típico y la influencia que sobre la estructura tiene el considerar los efectos P- $\Delta$ cuando ésta se somete a registros acelerométricos. El factor de escala de las aceleraciones de los registros utilizados $I$ toma el valor $I_{c}$ cuando induce inestabilidad dinámica al sistema.

Para detectar con mayor seguridad el valor de $C c$, se evalúan los desplazamientos máximos con efectos P- $\Delta$ en relación a dichos valores obtenidos sin considerar los efectos mencionados. El efecto que la carga gravitacional tiene sobre la estructura es notorio cuando el coeficiente de diseño sísmico se acerca al coeficiente de colapso ya que la relación antes mencionada comienza a incrementarse rápidamente hasta que las amplificaciones del desplazamiento por efecto P- $\Delta$ llegan a crecer asintóticamente.

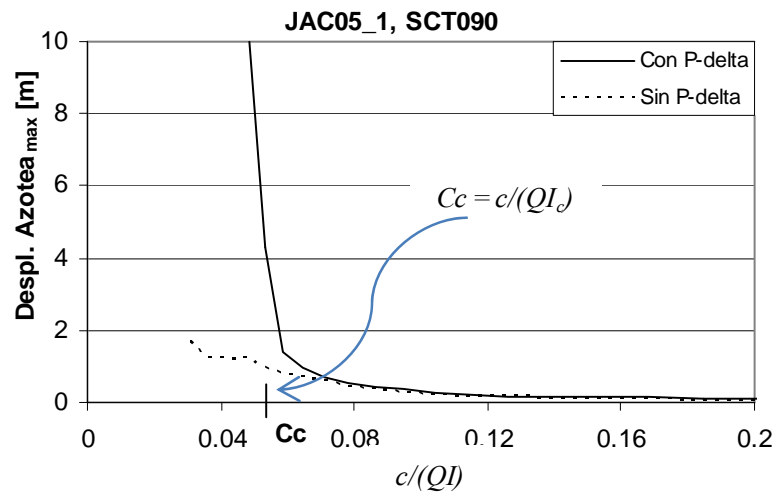

Figura 11. Coeficiente de colapso dinámico $(C c)$ para la estructura JAC05_1 sometida a un análisis dinámico (registro SCT090)

En la figura 12 se puede ver un grupo de curvas típicas para un conjunto de estructuras (submodelos del marco de referencia OBE23) sometidas a análisis dinámicos, se grafica el cociente del desplazamiento máximo de azotea con efecto P- $\Delta$ y sin efecto P- $\Delta$ (amplificación de desplazamiento máximo de azotea por efecto P- $\Delta, \alpha$ ) en función del correspondiente coeficiente de cortante basal de diseño sísmico, dichos valores estimados como se indicó en párrafos anteriores. El acelerograma utilizado para generar las curvas de la figura 12 es SCT090 el cual corresponde al sismo del 19 de septiembre de 1985 registrado en la estación de la Secretaría de Comunicaciones y Transportes E-O. Es claro como las amplificaciones en el desplazamiento por efecto P- $\Delta$ para ciertos coeficientes de diseño sísmico crecen irreversiblemente cuando este último coeficiente se acerca al coeficiente de colapso dinámico $(C c)$.

Para efectos de este estudio, la inestabilidad se presenta cuando el desplazamiento máximo de azotea es cuatro veces aquel obtenido de un análisis sin efectos P- $\Delta(\alpha=4)$, pues como se ve de la figura12 cuando se alcanza esta amplificación de desplazamientos por efectos P- $\Delta$ el sistema no recobra ya la estabilidad y se alcanza el estado límite de inestabilidad dinámica. Los análisis dinámicos se hacen 
Efecto de la transición al mecanismo plástico en la inestabilidad dinámica

con ayuda del programa de análisis estructural Drain 2Dx, en todos los casos se ha supuesto un porcentaje del amortiguamiento crítico del 2\% y no se considera endurecimiento por deformación del acero.

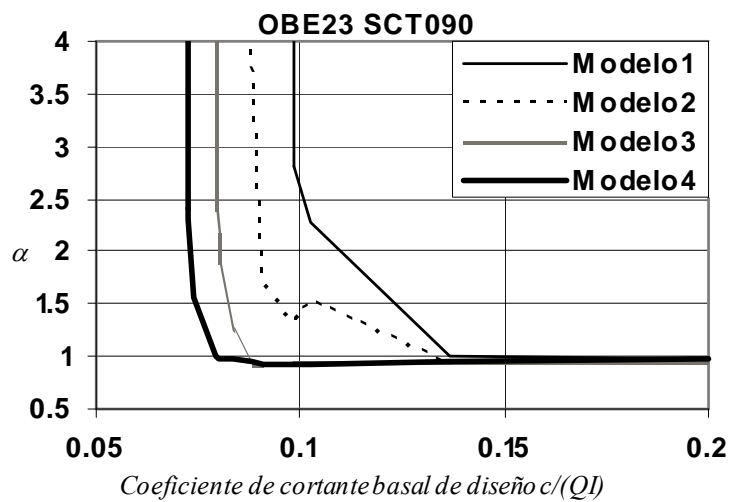

Figura 12. Amplificaciones por P- $\Delta$ para diferentes coeficientes basales de diseño

Tabla 5. Coeficientes de colapso para cada modelo en estudio

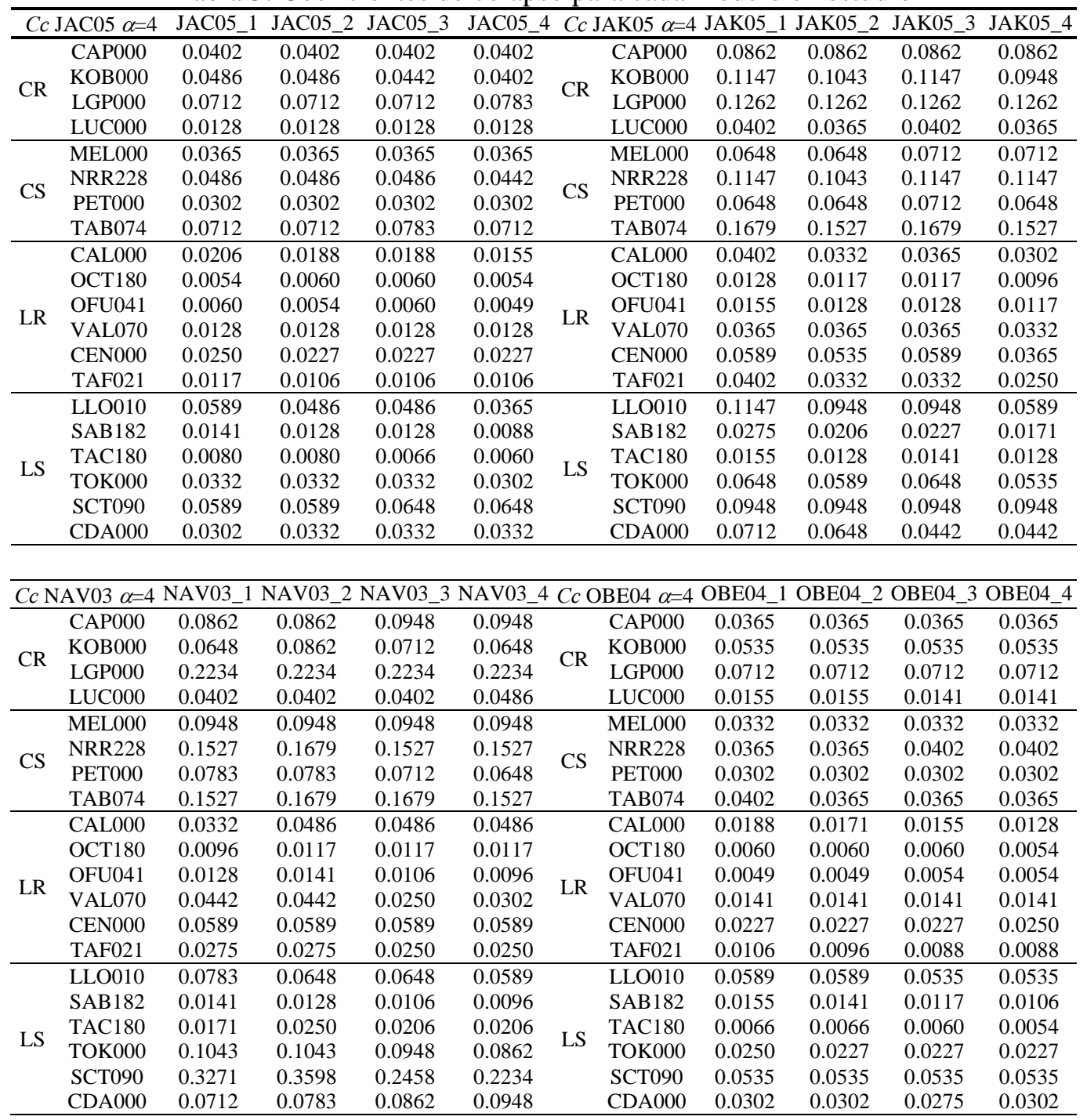


Gerardo Díaz Martínez y Emilio Sordo Zabay

Tabla 5. Coeficientes de colapso para cada modelo en estudio. Continuación

\begin{tabular}{|c|c|c|c|c|c|}
\hline$C_{c}$ & BE23 $\alpha=4$ & BE23_ & DBE23_ & OBE23. & BE23_4 \\
\hline & CAP000 & 0.3271 & 0.3271 & 0.3271 & 0.3271 \\
\hline$C R$ & КОВ000 & 0.4789 & 0.4789 & 0.4789 & 0.4789 \\
\hline Un & LGP000 & 0.6376 & 0.7013 & 0.7013 & 0.7013 \\
\hline & LUC000 & 0.1679 & 0.1679 & 0.1679 & 0.1679 \\
\hline & MEL000 & 0.2974 & 0.3271 & 0.3271 & 0.3271 \\
\hline CS & NRR228 & 0.4354 & 0.4354 & 0.4354 & 0.4354 \\
\hline CS & PET000 & 0.2458 & 0.2458 & 0.2703 & 0.2458 \\
\hline & ТAB074 & 0.7013 & 0.7013 & 0.7013 & 0.6376 \\
\hline & CAL000 & 0.2234 & 0.2234 & 0.2031 & 0.1847 \\
\hline & OCT180 & 0.0535 & 0.0589 & 0.0589 & 0.0589 \\
\hline IR & OFU041 & 0.0535 & 0.0535 & 0.0486 & 0.0486 \\
\hline $\mathrm{L}$ & VAL070 & 0.1679 & 0.1679 & 0.1527 & 0.1388 \\
\hline & CEN000 & 0.2974 & 0.2703 & 0.2703 & 0.2234 \\
\hline & TAF021 & 0.1262 & 0.1262 & 0.1262 & 0.1147 \\
\hline & LLO010 & 0.3598 & 0.2703 & 0.3271 & 0.2974 \\
\hline & SAB182 & 0.0712 & 0.0712 & 0.0712 & 0.0712 \\
\hline & TAC180 & 0.0862 & 0.0712 & 0.0712 & 0.0648 \\
\hline LS & TOK000 & 0.2703 & 0.2458 & 0.2458 & 0.2458 \\
\hline & SCT090 & 1.1293 & 1.0267 & 0.9333 & 0.8485 \\
\hline & CDA000 & 0.2703 & 0.2703 & 0.2703 & 0.2703 \\
\hline
\end{tabular}

Los resultados obtenidos del análisis descrito anteriormente (coeficientes de colapso dinámico) se muestran en la tabla 5 donde se han agrupado los submodelos de cada marco de referencia y los registros de acuerdo a la clasificación propuesta.

La tabla 6 muestra los coeficientes de colapso dinámico normalizados al valor máximo que toma éste entre submodelos para cada registro utilizado, se resaltan valores con diferencias mayores a un $20 \%$.

La información resumida en la tabla 6 se sintetiza en la tabla 7 en la que se presenta para cada marco de referencia el $C c$ menor de todos los submodelos normalizado al coeficiente mayor de todos ellos. Se resalta en la tabla cuáles son los valores que implican diferencias mayores a un $20 \%$, los cuales se podría afirmar presentan una variación importante en el coeficiente de colapso por inestabilidad dinámica.

Tabla 6. Coeficientes de colapso normalizados al valor máximo de cada submodelo

\begin{tabular}{|c|c|c|c|c|c|c|c|c|c|c|c|}
\hline \multicolumn{2}{|c|}{$C c / C c_{\max } \alpha=4$} & AC05_1 & JAC05_2 & JAC05_3 & JAC05_4 & c/ & $C c_{\max } \alpha=4$ & $\mathrm{AK} 05$ & IAK05_2 & JAK05_3 & JAK05_4 \\
\hline \multirow{4}{*}{ CR } & CAP000 & 1.0000 & 1.0000 & 1.0000 & 1.0000 & \multirow{4}{*}{ CR } & CAP000 & 1.0000 & 1.0000 & 1.0000 & 1.0000 \\
\hline & КОВ000 & 1.0000 & 1.0000 & 0.9091 & 0.8265 & & КОВ000 & 1.0000 & 0.9093 & 1.0000 & .8266 \\
\hline & LGP000 & 0.9092 & 0.9092 & 0.9092 & 1.0000 & & LGP000 & 1.0000 & 1.0000 & 1.0000 & .0000 \\
\hline & LUC000 & 1.0000 & 1.0000 & 1.0000 & 1.0000 & & LUC000 & 1.0000 & 0.9090 & 1.0000 & .9090 \\
\hline \multirow{4}{*}{ CS } & MEL & & (n) & & 1.00 & \multirow{4}{*}{ CS } & & 90 & 0 & & 000 \\
\hline & NRR & & 00 & & 0.9091 & & NRI & & & & 00 \\
\hline & РET000 & 00 & 1.0000 & 1.0000 & 1.0000 & & $\mathrm{PE}$ & 0.9090 & 90 & 00 & 090 \\
\hline & TAB & 9092 & 92 & 0 & 0.9092 & & $\mathrm{TA}$ & 1.0 & 91 & 0 & 091 \\
\hline \multirow{6}{*}{ LR } & & & 094 & & 7513 & \multirow{6}{*}{ LR } & 0 & 1.0000 & 64 & 90 & 12 \\
\hline & OCT180 & & 00 & & 9080 & & 80 & & 94 & 94 & .7510 \\
\hline & OFU0 & 0000 & 9080 & 000 & 0.8261 & & $\mathrm{OF}$ & 1.0000 & 0.8265 & 0.8265 & 0.7516 \\
\hline & VAL070 & 1.0000 & 1.0000 & 1.0000 & 1.0000 & & VAL070 & 1.0 & & 000 & 091 \\
\hline & & & & & 91 & & & & & & 208 \\
\hline & & & & & 0.9090 & & & & & & 209 \\
\hline \multirow{6}{*}{ LS } & & & & & & \multirow{6}{*}{ LS } & & & & & 33 \\
\hline & & & 2 & & 10 & & & & & & \\
\hline & TAC & & 00 & & 0.7522 & & TAC & 1.0000 & 0.8265 & 0.9090 & 0.8265 \\
\hline & 0 & & 0 & 0 & 0.9091 & & & & & 1.0000 & .8263 \\
\hline & SCT & 0 & 0.9090 & 1.0000 & 1.0000 & & SCT090 & 1.0000 & 1.0000 & 1.0000 & 1.0000 \\
\hline & CDA000 & 0.9091 & 1.0000 & 1.0000 & 1.0000 & & CDA000 & 1.0000 & 0.9090 & 0.6208 & 0.6208 \\
\hline
\end{tabular}


Tabla 6. Coeficientes de colapso normalizados al valor máximo de cada submodelo. Continuación

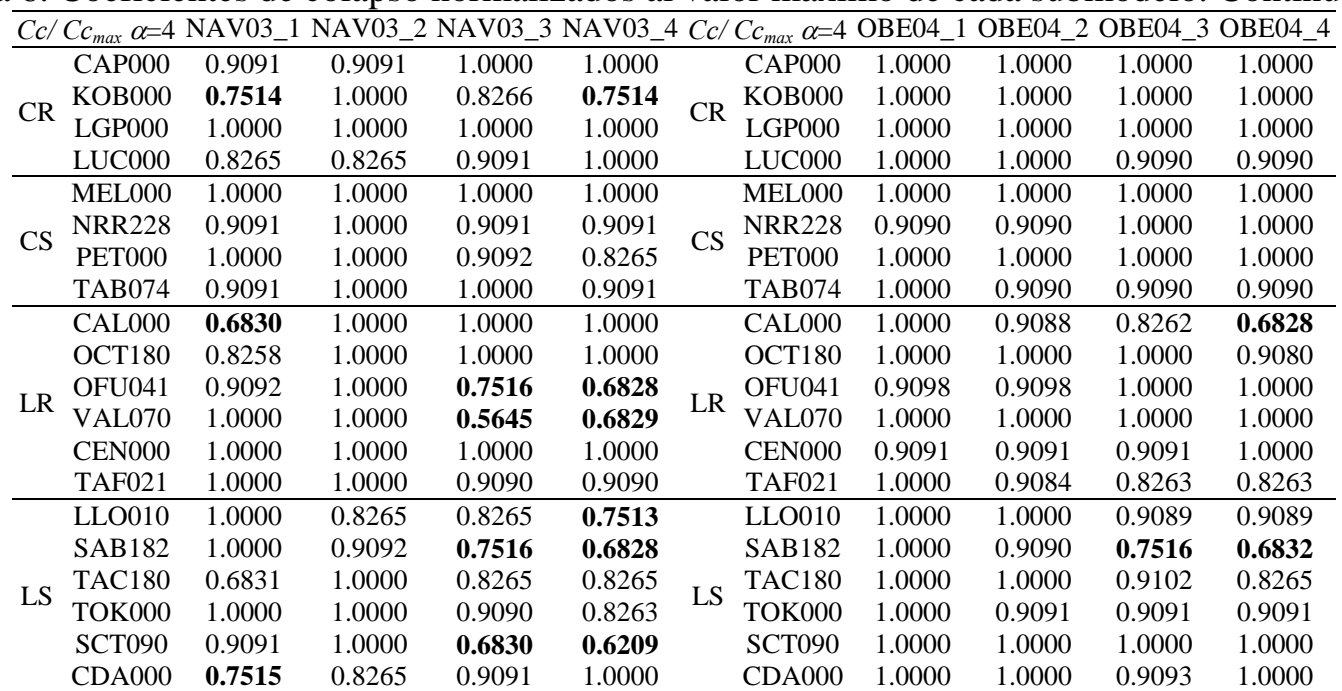

\begin{tabular}{|c|c|c|c|c|c|}
\hline \multicolumn{6}{|c|}{$C c / C c_{\max } \alpha=4$ OBE23_1 OBE23_2 OBE23_3 OBE23_4 } \\
\hline \multirow{4}{*}{ CR } & CAP000 & 1.0000 & 1.0000 & 1.0000 & 1.0000 \\
\hline & KOB000 & 1.0000 & 1.0000 & 1.0000 & 1.0000 \\
\hline & LGP000 & 0.9091 & 1.0000 & 1.0000 & 1.0000 \\
\hline & LUC000 & 1.0000 & 0 & & 000 \\
\hline \multirow{4}{*}{ CS } & ME & & & & 000 \\
\hline & NRR & & 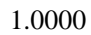 & & 000 \\
\hline & PET & 1.9 & 0.9091 & 1.0000 & 9091 \\
\hline & $\mathrm{TAI}$ & 1.0000 & 1.0000 & 1.0000 & .9091 \\
\hline \multirow{6}{*}{ LR } & CA & 1.0 & 00 & 0.9091 & .8266 \\
\hline & OC & 0.9089 & 1.0000 & & .0000 \\
\hline & OFU041 & 1.0000 & 1.0000 & 0.9092 & 0.9092 \\
\hline & VAL070 & 1.0000 & & & 0.8265 \\
\hline & CEN & & & & 0.7512 \\
\hline & & & & & 0.9089 \\
\hline \multirow{6}{*}{ LS } & & & & & 0.8265 \\
\hline & & & & & 1.0000 \\
\hline & TAC180 & 1.0000 & 0.8266 & 0.8266 & 0.7514 \\
\hline & TOK000 & 1.0000 & 0.9091 & 0.9091 & 0.9091 \\
\hline & SCI & 1.0000 & 0.9091 & 0.8264 & 0.7514 \\
\hline & CDA000 & 1.0000 & 1.0000 & 1.0000 & 1.0000 \\
\hline
\end{tabular}

De las tablas anteriores se puede observar que, para sismos cercanos epicentralmente (CR, CS tabla 4) no parece haber influencia de la forma en que se desarrolla el mecanismo plástico sobre los valores que toma el coeficiente de colapso. Sin embargo, para la mayoría de sismos de generación lejana (LR, LS tabla 4) existen diferencias importantes en el coeficiente de colapso dependiendo de la forma en la que se produzca la transición del estado elástico al mecanismo plástico. Lo anterior dado que los menores coeficientes de colapso normalizados (ver resumen de valores en la tabla 7) se dan precisamente para registros clasificados como LR y LS según la tabla 4. En particular, se observa de las tablas 5 a 7 que los modelos JAK05 y NAV03 son los que presentan mayores diferencias del $C c$ entre submodelos, en forma consistente con el hecho de ser éstos los marcos en los que se observan diferencias más notables en el comportamiento de las curvas de segundo ciclo de empujón estático con efectos P- $\Delta$ asociadas a los diferentes submodelos (ver figura 8). En el modelo JAC05 pese a existir importantes diferencias en las curvas de segundo ciclo, el coeficiente de estabilidad es relativamente pequeño, por lo que disminuyen las diferencias entre los coeficientes de colapso de los diferentes submodelos, debido a un menor efecto negativo de la carga gravitacional. 
Gerardo Díaz Martínez y Emilio Sordo Zabay

Tabla 7. Valor mínimo del coeficiente de colapso normalizado al valor máximo de cada submodelo

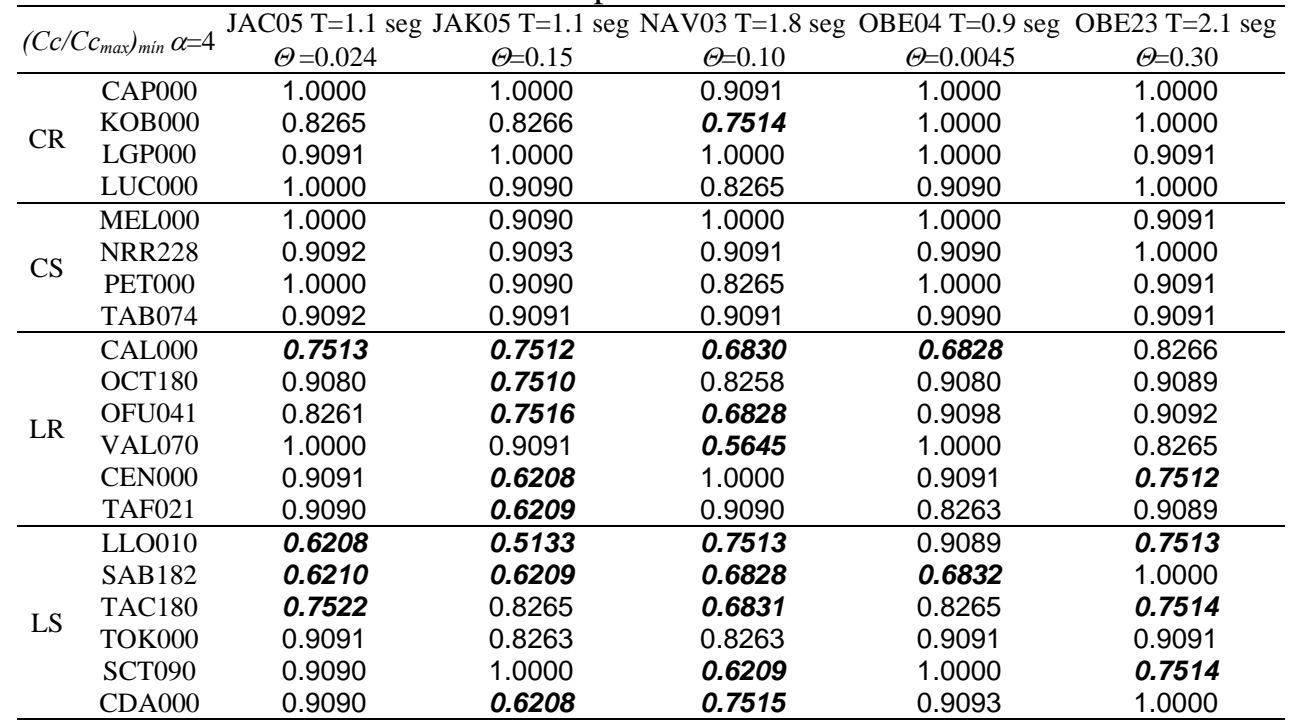

Del análisis de las tablas 3 y 7 se puede comentar que existe relación entre las diferencias encontradas en el trabajo de segundo ciclo de empujón estático para cada marco de referencia y las diferencias que existen en los coeficientes de colapso entre submodelos de un mismo marco de referencia. Los marcos de referencia NAV03 y JAK05 son los que muestran mayores diferencias en cuanto al trabajo de segundo ciclo de cada submodelo, consistentemente con el hecho de que son estos marcos los que presentan las diferencias más grandes en cuanto al coeficiente de colapso se refiere, por otro lado, el marco de referencia OBE23 muestra diferencias casi nulas para ambos casos, trabajo estático de segundo ciclo y coeficientes de colapso. Lo anterior se relaciona también con el hecho de que los primeros dos marcos mencionados presentan coeficientes de estabilidad mayores que el marco OBE23, con lo que se observa que a mayor efecto negativo de la carga gravitacional, las diferencias en el trabajo producido en segundos ciclos de empujón estático son indicadores de las variaciones del $C c$ entre marcos con las mismas propiedades elásticas, cortante resistente y forma del mecanismo de falla.

De lo anterior se podría afirmar que comparando dos estructuras con las mismas propiedades elásticas, cortante basal último resistente y forma del mecanismo, pero con diferente transición entre el estado elástico y el plástico sometidas a análisis dinámicos; aquella con transición al mecanismo de falla mas paulatina y con trabajo estático de segundo ciclo menor $(\mathrm{Wm})$ tendrá un coeficiente de colapso por inestabilidad dinámica menor (factor de escala de aceleraciones más alto).

\section{SISTEMAS DE UN GRADO DE LIBERTAD CON COMPORTAMIENTO HISTERÉTICO TRILINEAL}

\section{Parámetros asociados a los Sistemas de un Grado de Libertad Trilineales}

Como segunda parte de este trabajo se estudia el comportamiento de sistemas de un grado de libertad trilineales (SUGLT) para tratar de determinar qué parámetros influyen en el efecto de la transición elastoplástica sobre el coeficiente de colapso aquí representado por $c t_{l}$. La figura 13(a) muestra esquemáticamente una curva de capacidad con transición elastoplástica paulatina y pendiente negativa pronunciada debido a la acción de altos niveles de carga gravitacional; asimismo se presentan modelos 
bilineales y trilineales asociados a esta curva de capacidad. Se utilizan los modelos trilineales para modelar de manera más aproximada la transición del estado elástico al plástico de los sistemas en estudio y así determinar si existe influencia de esta transición en el coeficiente de colapso por inestabilidad dinámica. Los SUGLT que se estudian se caracterizan por los siguientes parámetros: $C_{y}$ coeficiente de fluencia del SUGLT, $\theta$ coeficiente de estabilidad del sistema, $\zeta$ porcentaje del amortiguamiento crítico, $\mu_{\mathrm{t}}$ ductilidad del tramo de transición, $T_{0}$ periodo inicial, tales parámetros se muestran en la figura 13(b).

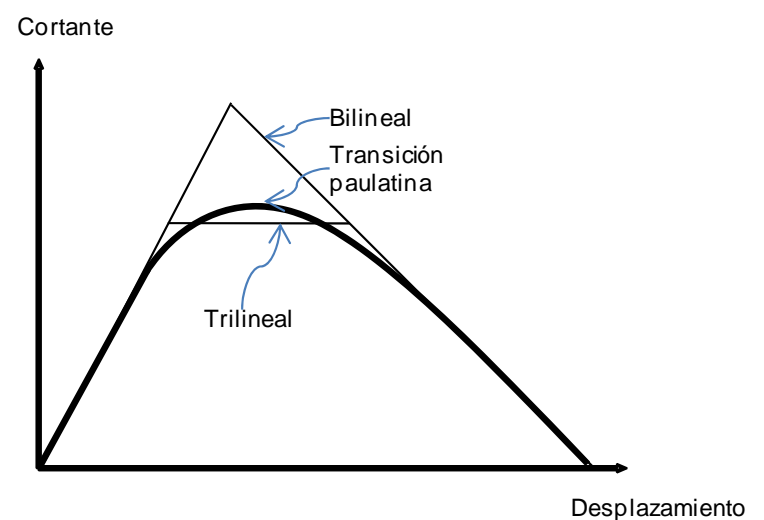

(a)

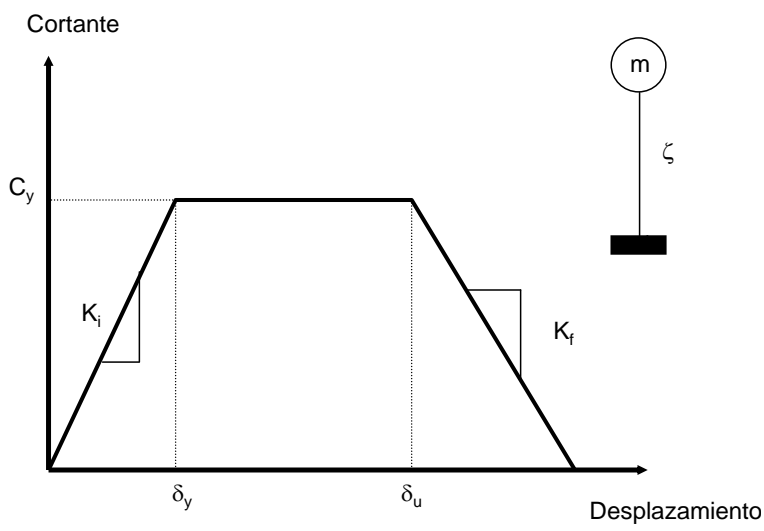

(b)

Figura 13. Características de los sistemas de un grado de libertad trilineales en estudio a) modelos bilineales y trilineales asociados a una curva de capacidad b) parámetros en estudio

Los parámetros mostrados en la figura 13 y mencionados en el párrafo precedente se definen por:

$K_{i}=\left(\frac{2 \pi}{T_{0}}\right)^{2}$

$K_{i} \quad$ rigidez de la primera rama del SUGLT

$T_{0} \quad$ periodo inicial del SUGLT

$\theta=\frac{K_{f}}{K_{i}}$

$\theta \quad$ coeficiente de estabilidad del SUGLT

$K_{f} \quad$ rigidez de la tercera rama del SUGLT

$\mu_{t}=\frac{\delta_{u}}{\delta_{y}}$

$\mu_{t} \quad$ ductilidad desarrollada por el SUGLT

$\delta_{u} \quad$ desplazamiento último del SUGLT

$\delta_{y} \quad$ desplazamiento de fluencia del SUGLT

La figura 13 (b) muestra esquemáticamente los parámetros antes definidos. 


\section{Análisis dinámico de los SUGLT}

Se efectúa un análisis paramétrico de SUGLT generados para el estudio, tales sistemas se someten a análisis dinámicos para determinar parámetros asociados a su respuesta ante solicitaciones sísmicas (coeficiente de fluencia asociado al desplazamiento de colapso, trabajo monotónico máximo y energía total máxima) dichos parámetros se definen más adelante.

Los análisis dinámicos se hacen con ayuda del programa TRILIN, el cual efectúa análisis no lineales paso a paso de sistemas con ciclo histerético trilineal mediante el método de la aceleración constante promedio y considerando los algoritmos de localización de eventos (descarga y fluencia dentro del paso de integración) propuestos por Bernal (1991), cuidando diversos aspectos relacionados con la precisión de los cálculos para evitar que inestabilidades numéricas puedan esconder el fenómeno en estudio. El programa de análisis lineal paso a paso se calibró con resultados obtenidos del programa DRAIN 2DX (Prakash et al., 1993).

Los registros acelerográficos utilizados en el análisis dinámico de sistemas de un grado de libertad con ciclo histerético trilineal son aquellos descritos en la sección correspondiente a marcos planos.

Para el análisis paramétrico de los SUGLT se utilizan los siguientes valores de los parámetros en estudio. $\mu_{t}=1,2,3,4$ y $5, \xi=0.02,0.05, T_{0}=$ de 0.4 a 4.83 segundos, $\theta=\theta_{\min }, \theta_{\max }$ donde:

$\theta_{\min }=0.012 T_{0}^{0.64}$

Coeficiente de estabilidad mínimo, el cual le correspondería a una estructura con mecanismo de falla global donde el efecto de la carga gravitacional, por ser ésta moderada no desestabiliza de manera importante al sistema (Bernal, 1992).

$\theta_{\max }=0.082 T_{0}^{1.44}$

Coeficiente de estabilidad máximo, el cual corresponde a un valor crítico del coeficiente de estabilidad y que se asocia a un sistema estructural que presenta falla de piso suave donde el efecto desestabilizador de la carga gravitacional es muy grande (Bernal, 1992).

\section{Espectros de Resistencia de Colapso}

Como primera parte del estudio de sistemas de un grado de libertad trilineales, se obtienen espectros de resistencia de colapso (espectros de colapso), en los cuales se obtiene el coeficiente de cortante basal $\left(c t_{1}\right)$ para el cual durante la respuesta dinámica se alcanza cierto nivel de desplazamiento llamado desplazamiento de colapso (este coeficiente se corresponde con el coeficiente de colapso estudiado antes ver figura 14a). Para tal efecto, se realiza un proceso iterativo con el programa TRILIN mediante el cual se reduce paulatinamente el coeficiente de fluencia del SUGLT hasta que el sistema alcanza el desplazamiento de colapso $\left(\delta_{c}\right)$ durante su respuesta dinámica, definido éste como el desplazamiento para el cual el cortante en el sistema es cero en una curva cortante $v s$. desplazamiento (ver figura 14a).

El proceso iterativo para la generación de espectros de resistencia de colapso se sintetiza en los siguientes pasos. 
1. El proceso inicia proponiendo los parámetros antes definidos (ductilidad, porcentaje del amortiguamiento crítico, periodo del SUGLT y coeficiente de estabilidad) y un cortante de fluencia muy grande que asegure comportamiento elástico durante la respuesta del sistema. Del análisis dinámico se obtiene el desplazamiento máximo del sistema, el cual mediante la rigidez inicial se asocia a un nuevo coeficiente de fluencia (con este valor de $C_{y}$ reducido en un $1 \%$ se asegura que en un nuevo análisis el sistema rebasará el desplazamiento de fluencia).

2. Con el nuevo valor de $C_{y}$ se calcula el valor del desplazamiento de colapso y se realiza una vez más el análisis, se compara el valor de la respuesta máxima en valor absoluto con el desplazamiento de colapso, si de esta comparación resulta que la respuesta máxima es mayor o igual al desplazamiento de colapso el proceso se detiene y se denomina el valor del coeficiente de fluencia como $c t_{1}$ (coeficiente de fluencia asociado al desplazamiento de colapso).

3. Si el valor de la respuesta máxima del sistema resulta ser menor que el desplazamiento de colapso el valor del coeficiente de fluencia se reduce en $1 \%$ y se regresa al paso 2.

El proceso iterativo se repite para generar los correspondientes espectros de resistencia de colapso. Los espectros de colapso obtenidos se normalizan por el correspondiente espectro de resistencia de colapso de SUGLT con ductilidad de transición igual a uno (sistema bilineal). Los espectros se agrupan de acuerdo al tipo de suelo y a la distancia de la estación del registro al epicentro del mismo.

\section{Espectros de Trabajo Monotónico Máximo}

Se obtienen también espectros del trabajo monotónico máximo $(W c)$, es decir, se obtiene el trabajo monotónico máximo del SUGLT cuando durante la respuesta el desplazamiento máximo es igual o mayor al desplazamiento de colapso. Los espectros de trabajo monotónico máximo se normalizan al espectro correspondiente del SUGLT con ductilidad transicional igual a uno, y se presenta en raíz cuadrada, debido a que existe una proporcionalidad entre $W c$ y la resistencia elevada al cuadrado.

El trabajo monotónico máximo se define como el área bajo la curva cortante desplazamiento del SUGLT, como lo ilustra la figura 14b, $c t_{1}$ como se mencionó anteriormente, es el coeficiente de fluencia asociado al desplazamiento de colapso, el área sombreada corresponde al trabajo monotónico máximo asociado a cada caso. Los espectros así definidos se muestran en su totalidad en Díaz (2005). El análisis de resultados se presenta en secciones posteriores.
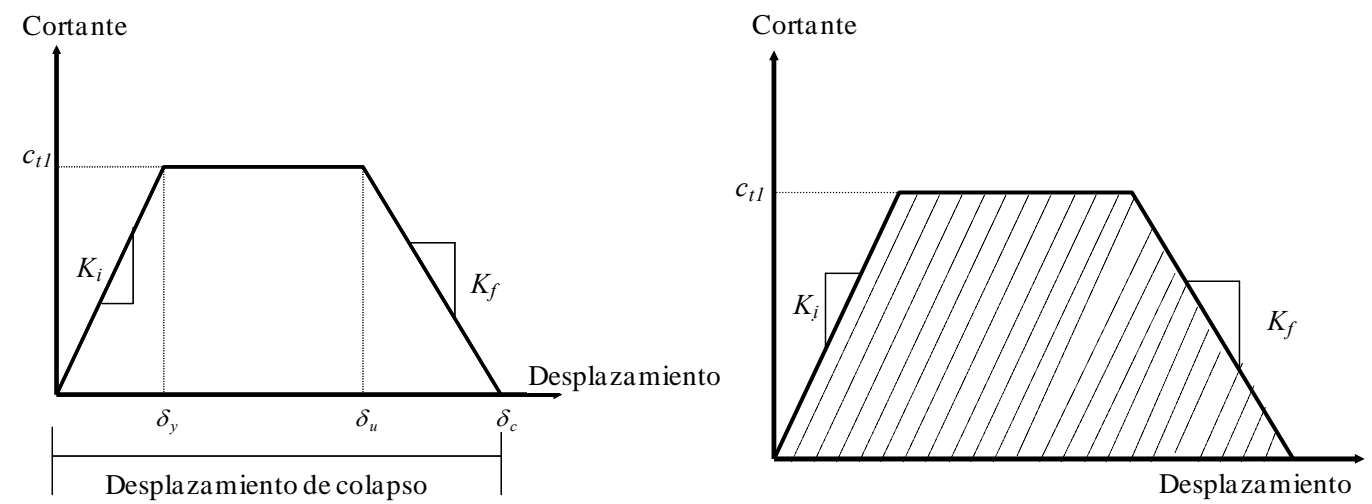

Figura 14. Definición del: a) desplazamiento de colapso, b) Trabajo monotónico máximo de los SUGLT 


\section{Espectros de Energía Total Máxima de Colapso de los SUGLT}

Se generan espectros de la energía total máxima $(E t m)$ disipada por el sistema en la respuesta asociada al umbral de la inestabilidad dinámica. Los espectros se construyen con la raíz cuadrada de la energía total máxima de los SUGLT para ductilidades transicionales de uno, dos, tres, cuatro y cinco, normalizadas a la raíz cuadrada de la energía total máxima del SUGLT con ductilidad transicional igual a uno (sistema bilineal).

La energía total máxima disipada durante la respuesta dinámica por los SUGLT queda definida por la siguiente expresión

$$
E t m=E c+E h+E a
$$

donde

$E c$ es la energía cinética disipada, $E h$ es la energía histerética disipada y $E a$ es la energía de amortiguamiento disipada.

Las figuras correspondientes a los espectros de energía total máxima para los sismos en estudio y para los diferentes parámetros considerados se muestran en su totalidad en Díaz (2005). El análisis de resultados se presenta en la sección siguiente.

\section{Sistemas de un Grado de Libertad Trilineales. Análisis de resultados}

De los espectros generados en el trabajo se puede sintetizar que las diferencias entre los parámetros normalizados (coeficiente de fluencia asociado al desplazamiento de colapso, trabajo monotónico de colapso y energía total máxima), en lo general crecen con el periodo y se presentan mayores diferencias en los casos en que los espectros se generan para el coeficiente de estabilidad máximo que para el mínimo como era de esperarse dado el mayor efecto desestabilizante de la carga gravitacional. Estas afirmaciones se ilustran en la figura 15 para el caso del coeficiente de fluencia asociado al desplazamiento de colapso, donde a la izquierda se muestran dos ejemplos para coeficiente de estabilidad mínimo y se comparan con los respectivos espectros generados para coeficiente de estabilidad máximo a la derecha en la figura. Mientras mayor es el coeficiente de estabilidad se observa una mayor variación en el coeficiente de colapso normalizado.

Una observación que resulta de la figura 16 es que el fenómeno de la inestabilidad dinámica parece no relacionarse con el porcentaje del amortiguamiento crítico, por lo menos para los dos valores estudiados en el presente trabajo ( $2 \%$ a la izquierda en la figura y $5 \%$ a la derecha). Como ejemplo, para espectros del mismo registro en estudio (en este caso se comparan los espectros correspondiente al registro NRR228 y al registro LLO010) y que comparten el coeficiente de estabilidad (mínimo y máximo respectivamente), solamente con diferente porcentaje de amortiguamiento crítico ( $2 \%$ y $5 \%$ para cada registro) no se aprecian diferencias significativas de la comparación de los espectros de la derecha con los de la izquierda en la figura 16. En el primer caso (registro NRR 228), no existen para ninguno de los dos porcentajes de amortiguamiento diferencias acentuadas entre los espectros por el distinto grado de amortiguamiento. En el segundo caso (LLO010), aunque para cada espectro se pueden observar diferencias para los coeficientes normalizados para diferentes ductilidades estas diferencias parecen no modificarse de un espectro a otro, por lo que otra vez el grado de amortiguamiento no modifica las tendencias observadas en los espectros. 
La figura 17 muestra una comparación de espectros de resistencia de colapso, trabajo monotónico de colapso y energía total máxima de colapso. En general puede verse que mientras crece la ductilidad de transición los resultados se alejan más de aquellos del modelo bilineal.
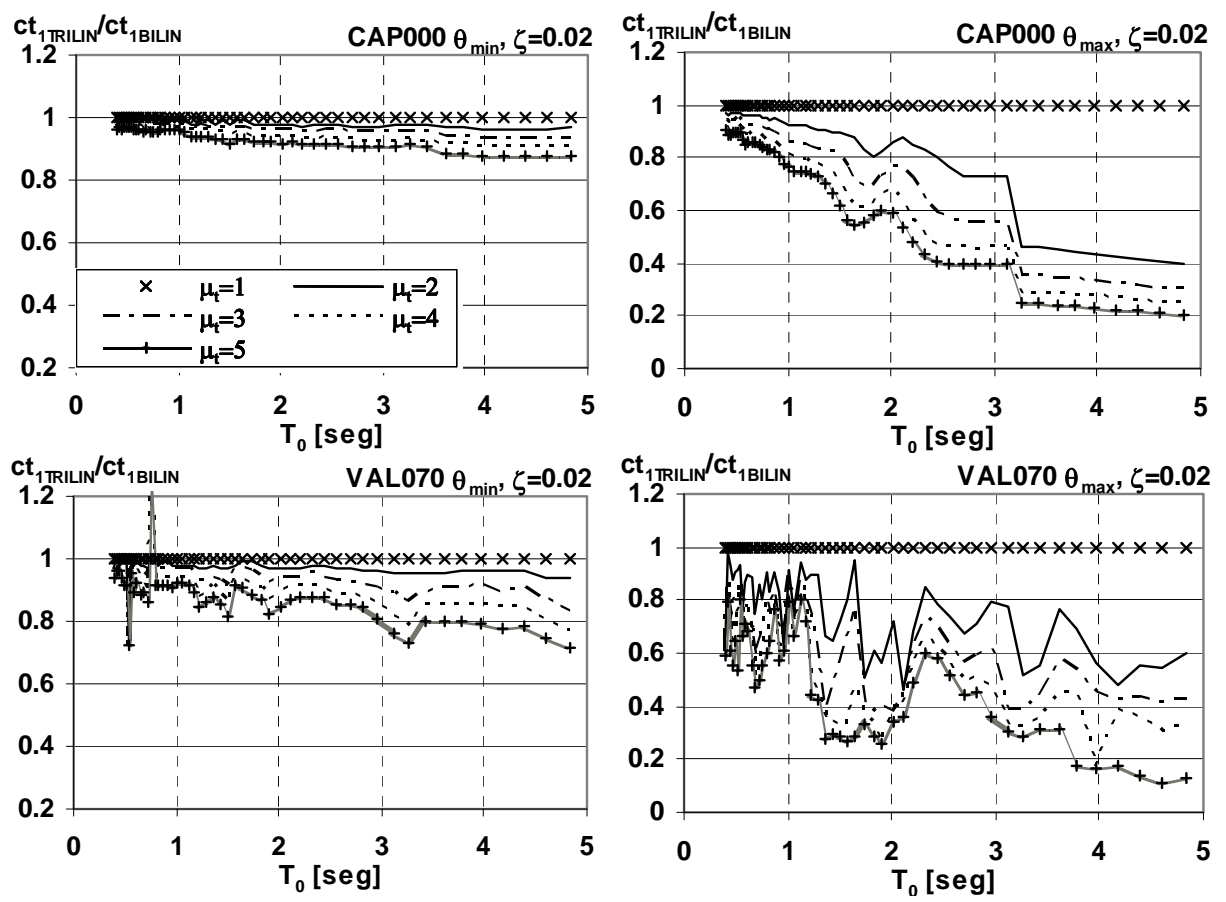

Figura 15. Resistencia de colapso para dos registros utilizados en el estudio, se muestra la diferencia entre el coeficiente de estabilidad mínimo y máximo
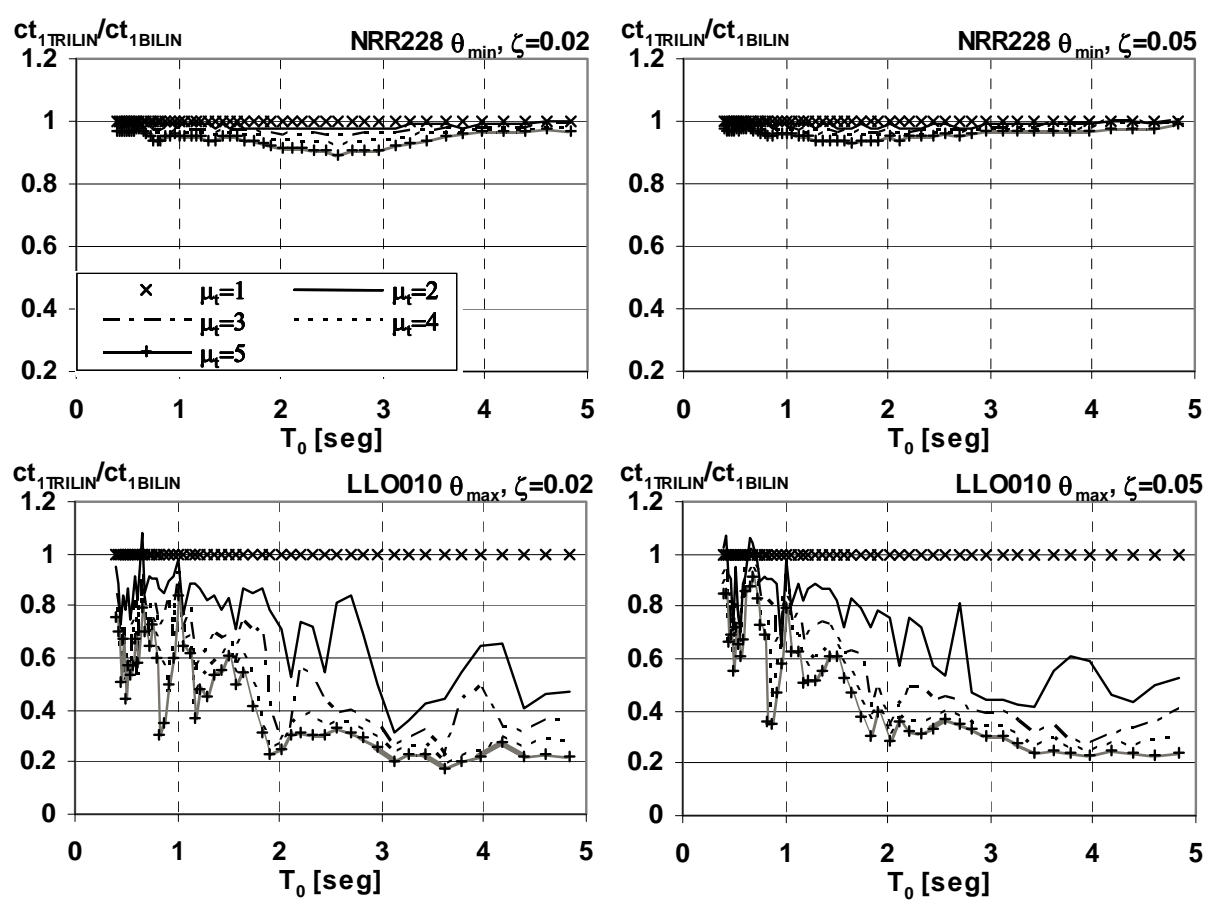

Figura 16. Resistencia de colapso para dos registros utilizados en el estudio, se muestra la no dependencia del porcentaje del amortiguamiento crítico 

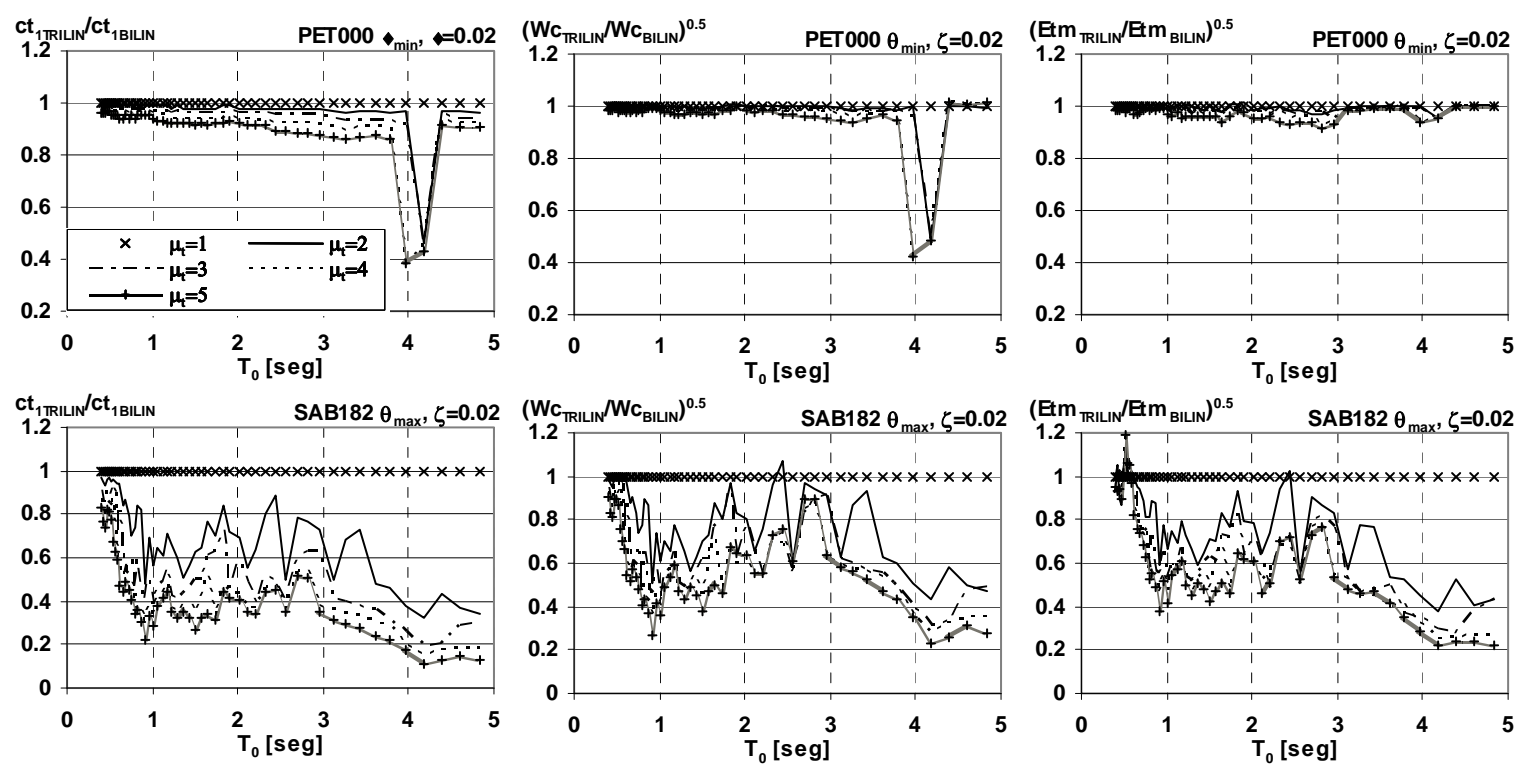

Figura 17. Espectros de resistencia, trabajo monotónico y energía total máxima de colapso para dos registros utilizados en el estudio

La figura 18 muestra espectros de energía total máxima de colapso (Etm) para 4 registros utilizados en el trabajo, coeficiente de estabilidad máximo y $\xi$ de $2 \%$. Un aspecto importante a destacar de la figura 18 es el hecho de que en los espectros de Etm las diferencias entre las curvas son por lo general menos pronunciadas y con menos diferencias abruptas (picos extremos en los espectros por ejemplo), lo que conduce a la idea de que los espectros de energía total máxima son un mejor indicador de las diferencias reales en el comportamiento de los SUGLT debidas a la ductilidad desarrollada por cada sistema.

Al igual que en el análisis de sistemas de múltiples grados de libertad, en el estudio de SUGLT se nota cierta diferencia entre los resultados de sismos de epicentro lejano y epicentro cercano, aunque no tan evidentes como en múltiples grados de libertad, para sismos de epicentro lejano las diferencias en el coeficiente de colapso tienden a ser mayores. En la figura 18 los espectros de energía total máxima de la izquierda corresponden a registros con epicentro cercano al sitio (CR, CS) y los de la derecha a epicentros lejanos (LR, LS), éstos clasificados de acuerdo a la tabla 4, se observa aquí la influencia de la distancia epicentral con valores que presentan mayores diferencias para la energía total máxima normalizada cuando los acelerogramas utilizados corresponden a aquellos clasificados como LR y LS.

De las figuras 15 a 18 se ve que para el coeficiente de estabilidad máximo se llegan a presentar diferencias grandes en los parámetros de respuesta (en el umbral de colapso) entre sistemas con diferentes ductilidades de transición, aun para sismos de generación cercana, este último hecho es atribuible a que el coeficiente de estabilidad máximo induce al sistema alta inestabilidad por efecto P- $\Delta$ caracterizada por una pendiente negativa pronunciada en la tercera rama del SUGLT. Se puede notar la consistencia en resultados entre los dos casos de estudio presentados en el trabajo, ya que las diferencias en el coeficiente de fluencia asociado al colapso, trabajo monotónico de colapso y energía total máxima son menores para sismos con epicentro cercano al sitio de registro (en el caso de SUGLT), este hecho se cumple también para los sistemas de múltiples grados de libertad para el coeficiente de colapso, especialmente para coeficiente de estabilidad pequeños (Díaz et. al. 2005). 
Por otro lado el efecto de la transición elastoplástica sobre el fenómeno de la inestabilidad dinámica resulta más evidente en los sistemas de un grado de libertad trilineales, por lo que debiera estudiarse con detalle una metodología apropiada para representar una estructura de múltiples grados de libertad con un sistema de un grado de libertad multilineal. Al respecto trabajos anteriores Díaz y Sordo (2005) y Zarfam y Mofid, (2011), han llegado a la misma conclusión sin proponer por ahora una reducción a sistemas de un grado de libertad trilineales equivalentes al sistema de múltiples grados de libertad.
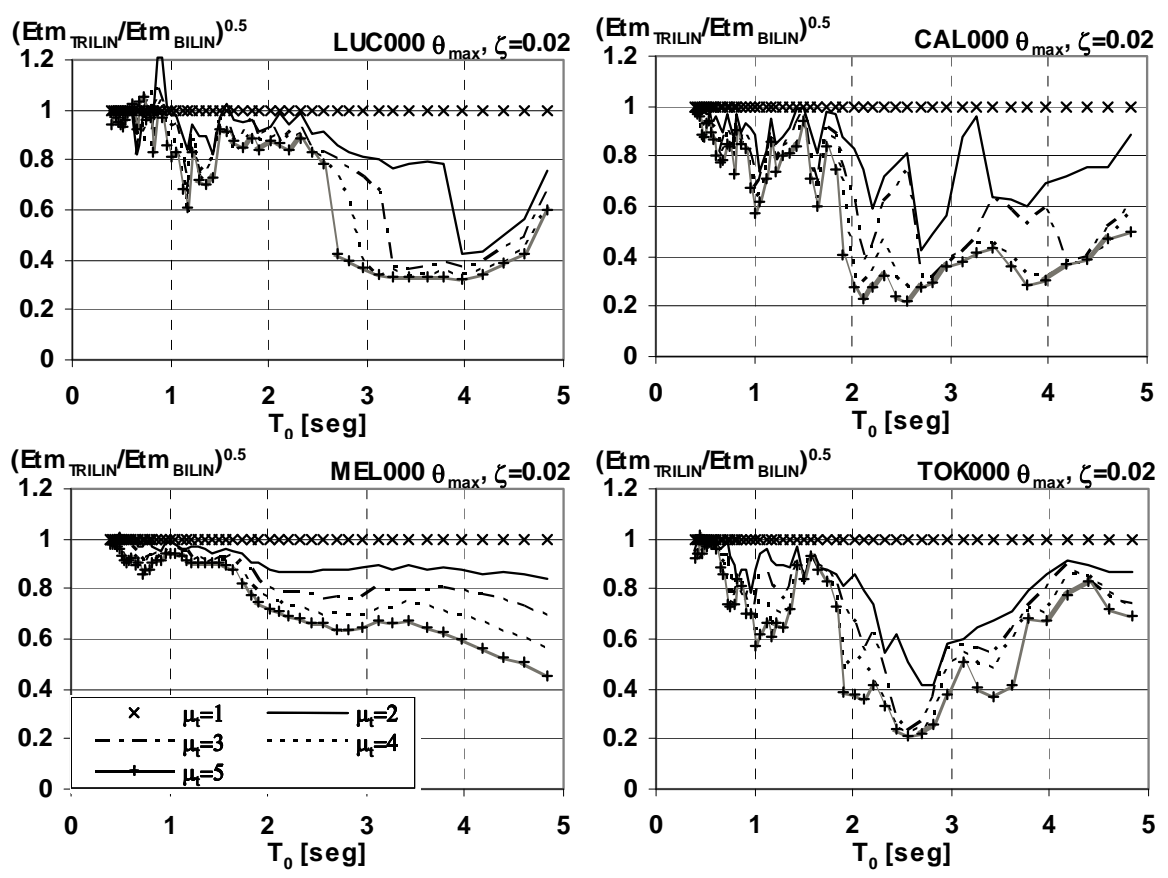

Figura 18. Espectros de energía total máxima de colapso para cuatro registros con diferente clasificación utilizados en el estudio

\section{CONCLUSIONES Y COMENTARIOS FINALES}

En el estudio se determinó si el proceso de transición del estado elástico al plástico en estructuras sometidas a análisis dinámicos tiene influencia sobre el coeficiente de colapso por inestabilidad dinámica; para ello, se analizaron un conjunto de marcos planos de acero y sistemas de un grado de libertad con comportamiento histerético trilineal, para establecer qué parámetros son los que influyen sobre el coeficiente de colapso por inestabilidad dinámica.

De los resultados presentados se pueden generar las siguientes afirmaciones.

El coeficiente de colapso llega a presentar significativas variaciones según el proceso particular de formación del mecanismo de colapso aún cuando los sistemas tengan similares características elásticas, cortante último y forma del mecanismo, especialmente cuando se someten a acelerogramas registrados a grandes distancias del epicentro.

De los resultados para marcos planos y SUGLT se puede afirmar que diseños basados en modelos elastoplásticos bilineales arrojan siempre diseños conservadores en cuanto a la inestabilidad dinámica se refiere, en comparación con sistemas estructurales con transición gradual del estado elástico al plástico. 
Una medida conservadora del coeficiente de colapso se puede obtener de sistemas de un grado de libertad equivalentes bilineales en términos generales.

Parece razonable realizar estimaciones de seguridad contra colapso por inestabilidad dinámica de estructuras mediante el análisis de sistemas de un grado de libertad bilineales basados en las características elásticas, el mecanismo de colapso y la resistencia basal última de las estructuras que representan, para movimientos del terreno catalogados aquí como cercanos al epicentro.

El porcentaje del amortiguamiento crítico no es un parámetro que afecte de manera importante el nivel de seguridad por inestabilidad dinámica, esto visto para el caso de SUGLT. Debe llevarse a cabo un estudio para marcos planos variando el nivel de amortiguamiento y verificar en su caso la veracidad de la afirmación anterior.

De los resultados obtenidos en el presente trabajo se puede afirmar que la transición del estado elástico al plástico de estructuras toma importancia en la variación del nivel de seguridad por inestabilidad dinámica, cuanto más crece el coeficiente de estabilidad de la estructura (este último relacionado estrechamente con el nivel de carga gravitacional presente). De acuerdo a los sistemas de un grado de libertad para periodos cortos los sistemas trilineales presentan pequeñas diferencias respecto a sistemas bilineales, estas diferencias crecen tanto con el periodo como con la ductilidad de transición.

\section{AGRADECIMIENTOS}

Los autores agradecen a la Dra. Consuelo Gómez, al Dr. Jaime de la Colina y al Dr. Alonso Gómez las observaciones vertidas en torno al trabajo aquí presentado, asimismo se agradece a los revisores del manuscrito sus comentarios y atinadas observaciones que ayudaron a enriquecerlo y mejorarlo. El primer autor desea agradecer al Consejo Nacional de Ciencia y Tecnología la beca otorgada para la realización de estudios de posgrado en la Universidad Autónoma Metropolitana unidad Azcapotzalco.

\section{REFERENCIAS}

Bernal, D (1991). "Locating events in step-by-step integration of the equations of motion", Journal of Structural Engineering, ASCE, Vol.117, No.2, pp. 530-545.

Bernal, D (1992), "Instability of buildings subjected to earthquakes”, Journal of Structural Engineering, $A S C E$, Vol. 118, No. 8, pp. 2239-2260.

Bernal, D (1998), “Instability of buildings during seismic response”, Engineering Structures, 20, 4-6, pp. 496-502.

Díaz, G y E Sordo, (2004) "El proceso de formación de mecanismos en la seguridad contra inestabilidad dinámica”, Memorias del XIV Congreso Nacional de Ingeniería Estructural, Acapulco, Guerrero, México.

Díaz, G, E Sordo y A Gómez, (2005) "Comportamiento de sistemas de un grado de libertad con ciclo histerético trilineal en el umbral de la inestabilidad dinámica”, Memorias del XV Congreso Nacional de Ingeniería Sísmica, México, D.F., México.

Díaz, G (2005) "Efecto de la transición al mecanismo plástico en la inestabilidad dinámica de marcos planos”, Tesis de Maestría, Posgrado en Ingeniería Estructural, Universidad Autónoma Metropolitana, Azcapotzalco, México DF. 
Díaz, G y E Sordo, (2008) "Escalamiento de registros del valle de México para la estimación de la seguridad contra inestabilidad dinámica”, Memorias del XVI Congreso Nacional de Ingeniería Estructural, Veracruz, Veracruz, México.

Iervolino I y A Cornell (2005), "Record selection for nonlinear seismic analysis of structures", Earthquake Spectra, Volumen 21, No. 3, agosto, pp. 685-713.

Elgamal, A, S Ashford y S Kramer, (1998), "1 st PEER workshop on characterization of special source effects”, Workshop Report, UCSD, Pacific Center for Earthquake Engineering Research, UC Berkeley.

Nakajima, A, Abe H y S Kuranishi (1990), "Effect of multiple collapse modes on dynamic failure of structures with structural instability", Proceedings of the Japan Society of Civil Engineers, No 416, pp. 13-23.

Prakash, V, G H Powell y S Campbell (1993), “Drain-2Dx base program description and user guide.”, Manual, University of California.

Sordo, E y D Bernal, (1993), "Influencia del mecanismo de falla en la seguridad contra colapso dinámico", Memorias del X Congreso Nacional de Ingeniería Sísmica, Puerto Vallarta, México.

Sordo, E y D Bernal, (1994), "Dynamic instability in three dimensional structures”, Report No. CE-94-14, Civil Engineering Department, Northeastern University, USA, 141 pages.

Sordo, E y D Bernal, (1996), “Dynamic instability phenomenon in bidirectional systems”, Proceedings of the Eleventh World Conference on Earthquake Engineering, Disc 1, Paper No. 327.

Sordo, E y G Díaz, (2003), “Importancia de la formación paulatina de mecanismos en la seguridad contra inestabilidad dinámica” Memorias del XIV Congreso Nacional de Ingeniería Sísmica, León, Guanajuato, México.

Sordo, E y I Félix, (2000), "Efecto de la orientación en la disipación energética de estructuras tridimensionales cercanas al colapso dinámico”, Memorias del XII Congreso Nacional de Ingeniería Sísmica, Morelia, México.

Tena, A (2007), Análisis de estructuras con métodos matriciales, Ed. Limusa México, pp. 353-356.

Vamvatsikos, D y C A Cornell, (2002), "Incremental dynamic analysis”, Earthquake Engineering and Structural Dynamics, Vol. 31, pp. 491-514.

Zarfam, P y M Mofid, (2011), “On the modal incremental dynamic analysis of reinforced concrete structures, using a trilinear idealization model”, Engineering Structures, Vol. 33, pp. 1117-1122. 